\title{
Synaptotagmin 1 oligomerization via the juxtamembrane linker regulates spontaneous and evoked neurotransmitter release
}

Kevin C. Courtney ${ }^{1,3}$, Yueqi $\mathrm{Li}^{1,2,3}$, Jason D. Vevea ${ }^{1,3}$, Zhenyong Wu${ }^{1}$, Zhao Zhang ${ }^{1}$ and Edwin R. 6 Chapman $^{1 *}$

${ }^{1}$ Howard Hughes Medical Institute and the Department of Neuroscience, University of Wisconsin, 1111 Highland Avenue, Madison, Wisconsin, 53705

${ }^{2}$ Center for Bioanalytical Chemistry, Hefei National Laboratory for Physical Sciences at the Microscale, Department of Chemistry, University of Science and Technology of China, Hefei, P. 11 R. China, 230027

$12{ }^{3}$ Equal contribution

*Corresponding author: E-mail: chapman@wisc.edu

\section{ABSTRACT}

Synaptotagmin-1 (syt1) is a $\mathrm{Ca}^{2+}$ sensor that regulates synaptic vesicle exocytosis. Cell-based experiments suggest that syt1 functions as a multimer, however biochemical and electron microscopy studies have yielded contradictory findings regarding putative self-association. Here, we performed dynamic light scattering on syt 1 in solution, followed by electron microscopy, and used atomic force microscopy to study syt1 self-association on supported lipid bilayers under aqueous conditions. Ring-like multimers were clearly observed. Multimerization was enhanced by $\mathrm{Ca}^{2+}$ and required anionic phospholipids. Large ring-like structures $(\sim 180 \mathrm{~nm})$ were reduced to smaller rings $(\sim 30 \mathrm{~nm})$ upon neutralization of a cluster of juxtamembrane lysine residues; further substitution of residues in the second C2-domain completely abolished self-association. When expressed in neurons, syt1 mutants with graded reductions in self-association activity exhibited concomitant reductions in: a) clamping spontaneous release, and b) triggering and synchronizing evoked release. Thus, the juxtamembrane linker of syt1 plays a crucial role in exocytosis by mediating multimerization. 
Synchronous release of neurotransmitters from presynaptic nerve terminals is triggered by the influx of $\mathrm{Ca}^{2+}$ ions via voltage gated $\mathrm{Ca}^{2+}$ channels $(1-3)$. The rise in $\left[\mathrm{Ca}^{2+}\right]_{i}$ is sensed by the synaptic vesicle (SV) protein, synaptotagmin 1 (syt1) $(4,5)$, which interacts with anionic phospholipids $(6-9)$ and SNARE proteins $(10,11)$ to trigger membrane fusion (12). Syt1 also plays additional roles in the regulation of the SV cycle, including: the inhibition of spontaneous release (clamping) (13-15), docking and priming $(16,17)$, and endocytosis (18-21). Although the importance of syt1 in the SV cycle is well established, whether and how syt1 performs each of these functions as a multimeric structure remains the subject of a long-standing debate. between several distinct mutant alleles of syt1 (13). This result strongly suggests that syt1 has separable functional domains and operates as a multimer in vivo. More recently, careful titration of mutant forms of syt1 (that were identified in human patients), in the presence of the wild-type protein, resulted in the potent dominant negative inhibition of SV exocytosis (22). This result further suggests that syt1 functions as an oligomer. However, the ability of syt1 to oligomerize, and the structure of these putative complexes, at both biochemical and ultrastructural levels, remains controversial.

SVs each contain approximately fifteen copies of syt1 (23), and multimerization of these monomers has been proposed to occur via two distinct mechanisms. The first proposed mode was constitutive and was mediated by determinants in the $\mathrm{N}$-terminal region of the protein, which comprises residues 1-142 (Fig. 1A). Using density gradient centrifugation, native syt1 was reported to assemble into $\mathrm{N}$-terminal-region mediated dimers (24) or tetramers in a nondenaturing detergent, CHAPS (4), but was also found to be largely monomeric in the absence of $\mathrm{Ca}^{2+}$ in Triton X-100 (25). N-terminal oligomerization was later refined in detergent-free conditions, 
transmembrane domain (TMD), and the first sixteen residues of the juxtamembrane linker (residues 80-142) between the TMD and the first $\mathrm{Ca}^{2+}$-sensing motif, C2A (26).

The second proposed mode of syt 1 oligomerization is mediated by the C-terminal $\mathrm{Ca}^{2+}$ sensing domain, $\mathrm{C} 2 \mathrm{~B}$ (Fig. 1A). The syt1 C2B domain has been implicated in numerous modes of oligomerization that range from $\mathrm{Ca}^{2+}$ triggered homo-dimerization to the formation of large multimeric complexes (27-29). Reported oligomerization motifs within C2B include residues K326/327 (27), F349 (28) and R398/399 (29). However, even though self-association of purified, recombinant $\mathrm{C} 2 \mathrm{~B}$ has been abundantly described $(25,30)$, this protein fragment was found to tightly bind bacterial contaminants (31). These contaminants alter the biochemical properties of $\mathrm{C} 2 \mathrm{~B}$, and thus complicate the interpretation of studies that did not include high salt washes and an RNAse/DNAse treatment to remove them during syt1 purification. Indeed, removal of these contaminants from $\mathrm{C} 2 \mathrm{~B}$ abolished its ability to self-associate in the presence or absence of $\mathrm{Ca}^{2+}$. However, in some studies, the addition of anionic phospholipid appeared to restore $\mathrm{Ca}^{2+}$-triggered oligomerization activity $(29,32-34)$.

Most recently, in a notable series of electron microscopy studies by Rothman, Krishnakumar, Volynski and colleagues, the cytoplasmic domain of syt1 formed ring-like oligomers on lipid monolayers when imaged via negative stain EM; these rings comprised >15 copies of syt1 (32). Interestingly, these rings were reported to be disrupted by $\mathrm{Ca}^{2+}$, and this dissolution was proposed to act as a mechanism to unclamp SV fusion (28, 32, 35-37). In contrast, other EM studies showed syt1 forms heptameric barrels in the presence of $\mathrm{Ca}^{2+}(34)$.

Due to the lack of consensus regarding the ability of syt1 to oligomerize, and the contradictory reports regarding the structure of these putative oligomers, we re-examined this question while adhering to physiological conditions that required minimal manipulation of the samples. To this end, we performed dynamic light scattering (DLS) on syt1 in solution, followed by electron microscopy (EM), and atomic force microscopy (AFM) imaging of syt1 on supported 
lipid bilayers (SLB). Together, these in vitro experiments served as a platform to screen for

81 putative oligomerization motifs that were then functionally tested in neurons. Importantly, these in

82 vitro experiments were conducted under aqueous conditions. Moreover, we used the complete

83 cytoplasmic domain of the protein (residues 80-421) (Fig. 1A). This contrasts with most of the

84 earlier in vitro work which utilized truncated forms of the cytoplasmic domain of syt1 (i.e. residues

$8596-421$ or $143-421$ ) that lacked either the highly cationic portion of the juxtamembrane region or

86 the entire juxtamembrane segment (Fig. 1A). By DLS and EM, we observed that anionic lipids

87 cause syt1(80-421) to assemble into large clusters. Moreover, AFM imaging found that syt1(80-

88 421) forms clusters, large ring-like structures, and large patches on phospholipid bilayers in an

89 anionic lipid dependent manner; formation of these multimeric structures on phospholipid bilayers

90 was enhanced by $\mathrm{Ca}^{2+}$. Mutagenesis revealed that lysine residues in the juxtamembrane linker

91 are crucially important for syt1 self-association. AFM imaging revealed that additional mutations,

92 in the C2B domain, further diminished the ability of syt1 to self-associate. In neurons, the ability

93 of syt1 to multimerize was well correlated with its ability to clamp spontaneous release and to

94 trigger and synchronize evoked release. Hence, the juxtamembrane linker enables syt1 to 95 assemble into a multimer, and this multimerization plays a key role in the regulation of SV 96 exocytosis.

\section{RESULTS}

The syt1 cytoplasmic domain forms multimeric structures, in solution, upon binding

\section{anionic phospholipids}

The structure of syt1 is depicted in Fig. 1A. The N-terminal region comprises a short

101 luminal domain, a single TMD and a juxtamembrane linker that is followed by the tandem C2-

102 domains (24). Within this juxtamembrane linker lies a sequence containing ten positively charged

103 residues directly after the TMD (Fig. 1A). A key feature of the experiments reported here is that

104 we used the intact cytoplasmic domain of syt1, residues $80-421$, which includes this cationic 
105 juxtamembrane segment. Again, this contrasts with the majority of published work describing syt1 106 biochemistry and oligomerization because those reports were based on shorter fragments 107 (residues $96-421$ or $143-421$, both lacking the polybasic region) (28-30, 32).

We first assessed the self-association properties of syt1 by performing DLS in aqueous media (Fig. 1B). Each of the two C2 domains of syt1 are approximately $2.5 \times 5 \mathrm{~nm}$ (SI Appendix, Fig. S1A). We reasoned that if syt1 assembled into a multimeric structure, the average hydrodynamic diameter of the pure protein should exceed these monomeric dimensions. When suspended in physiological, aqueous media, pure syt1(80-421) was found to have an average diameter of approximately $5 \mathrm{~nm}$, suggesting it is indeed monomeric (Fig. 1C). Syt1 is known to function by binding anionic lipids, namely phosphatidylserine (PS) and $\mathrm{PIP}_{2}$ (6-9). We therefore examined how anionic lipids would influence multimerization. Remarkably, we found that the addition of a soluble, short chain anionic lipid, 6:0 PS, caused syt1 to assemble into $\sim 250 \mathrm{~nm}$ structures (Fig. 1C). In contrast, syt1 remained monomeric in the presence of a non-acylated variant (phospho-serine) (SI Appendix, Fig. S1B) and 6:0 PS alone failed to generate detectable DLS signal (data not shown). This demonstrates that syt1 self-association is triggered upon binding anionic phospholipids, mediated by electrostatic and hydrophobic interactions. We then

121 proceeded to image the DLS samples by negative-stain EM. In line with the DLS, EM imaging 122 found large clusters in the WT syt1(80-421) + 6:0 PS sample, whereas protein alone and 6:0 PS 123 alone samples were devoid of these large structures (Fig. 1D). structures to exist in vivo. However, these findings show that the cytoplasmic domain of syt1 has the ability to self-associate in the presence of anionic lipids. As such, we reasoned that analyzing

127 the size of these supraphysiological multimeric structures, in conjunction with site-directed 128 mutagenesis, would enable us to map the determinants that mediate lipid-dependent homomeric 129 interactions under aqueous conditions. 


\section{Syt1 self-association persists after substitution of residues in the C2B domain}

To gain insight into the structural elements of syt1(80-421) that mediate self-association, we mutated a number of residues that have previously been reported to regulate syt1 oligomerization through the C2B domain and performed DLS analysis. These mutant forms of syt1 encompass: $\mathrm{K} 326,327 \mathrm{~A}(27,34)$, a positively charged region that is also responsible for $\mathrm{Ca}^{2+}$ independent $\mathrm{PIP}_{2}$ binding activity (7); F349A, which was reported to disrupt the ring structures observed by negative stain EM (28, 36); and R398,399Q, implicated in binding SNAREs and C2B self-association via back-to-back dimerization (29). All three sets of mutations failed to disrupt multimerization under our experimental conditions (Fig. 1C and Table 1).

\section{Lysine residues in the juxtamembrane linker of syt1 regulate self-association}

As outlined in the Introduction, syt1 was first thought to self-associate via determinants in the $\mathrm{N}$-terminus of the protein $(4,26)$. Within this putative oligomerization region, the juxtamembrane linker was shown to mediate $\mathrm{Ca}^{2+}$-independent interactions with membranes (38).

The juxtamembrane linker contains a segment (between residues 80-95) in which ten of the first sixteen residues after the transmembrane domain are lysines (Fig. 1A). This cationic region is poorly characterized as residues $80-95$ are commonly excluded from recombinant preparations of the syt1 soluble domain, perhaps due to increased difficultly in purification (see Methods). We hypothesized that these charged residues mediate interactions with anionic phospholipids to promote oligomerization $(4,26,38)$. To assess this possibility, we neutralized the juxtamembrane lysines (Juxta K) (SI Appendix, Fig. S2) and, again, performed DLS on syt1(80-421) with and without 6:0 PS. In contrast to WT protein, we found that 6:0 PS failed to trigger self-association of the Juxta $\mathrm{K}$ variant; Juxta $\mathrm{K}$ syt1 remained monomeric in solution (Fig. $1 \mathrm{C}$ and Table 1). This DLS result was further validated by EM imaging, which found that the Juxta $K+6: 0$ PS sample completely lacked large protein-lipid clusters (Fig. 1D). 


\section{The syt1 cytoplasmic domain forms multimeric structures on supported lipid bilayers}

Although DLS and EM analysis served as an efficient screen that enabled us to uncover Juxta $\mathrm{K}$ mediated syt 1 self-association, this system is accompanied by non-physiological caveats. To validate the DLS and EM results, we developed a second in vitro assay to examine if the syt1 cytoplasmic domain self-associates on the surface of phospholipid bilayers. For this, we generated SLBs and performed AFM imaging while aiming to mimic native, physiological conditions (Fig. 2A). This AFM strategy combines the distinct advantages of both DLS and EM by enabling high sensitivity experiments, with single particle resolution, in aqueous media. As preliminary test of our AFM approach, we incubated $1 \mu \mathrm{M}$ syt1(80-421) with a SLB (DOPC/DOPS/PIP $2,72: 25: 3$ ) for 6 hours in the absence of $\mathrm{Ca}^{2+}$. Under these aqueous conditions, we observed the formation of large numbers of ring-like structures formed by syt 1 on the lipid bilayer surface (SI Appendix, Fig. SA). A zoomed-in three-dimensional view of a representative structure is also shown (SI Appendix, Fig. S3B). Overall, the diameter of these structures (i.e. the distance between the two highest points on both sides in a cross-section) was commonly greater than $100 \mathrm{~nm}$, suggesting that they are formed by a high copy number of syt1(80-421).

Importantly, the syt 1 rings that we observed by AFM are filled with lipid in the center, as determined by lateral height profiles in conjunction with an analysis of surface roughness inside and outside the rings (SI Appendix, Fig. S3B and S3C). These findings indicate that these structures assemble on the surface of intact bilayers. We also occasionally observed structures that formed around defects (holes) in the SLB (SI Appendix, Fig. S4A). These structures resemble protein-decorated holes that form on SLBs after treatment with pore-forming proteins, such as Bax (Salvador-Gallego et al., 2016). However, since syt1(80-421) does not form large pores in bilayers (SI Appendix, Fig. S4B), we believe the effects of pore-forming proteins, like Bax, on SLBs are distinct from the ring-like structures that we observed. Indeed, upon further examination, we found that defects in the SLB are stable over time (SI Appendix, Fig. S4C), while the number 
of ring-like structures increase dramatically with time (SI Appendix, Fig. S4D). Hence, syt1 ring formation does not require membrane defects, and these ring-like structures represent bona fide syt1 multimers on the SLB surface. Notably, since the properties of the structures that associate around membrane defects are dominated by the size and shape of the defect itself, rather than the multimerization properties of syt1, we excluded structures with an interior hole or defect in the bilayer from all analyses.

\section{Syt1 self-association requires anionic phospholipids and is enhanced by $\mathrm{Ca}^{2+}$}

After establishing that circular multimerization (i.e. ring formation) on the bilayer surface was robust and reproducible by AFM imaging, we set the incubation time of syt1(80-421) with the SLB to 20 minutes in all subsequent trials. In the absence of $\mathrm{Ca}^{2+}(0.5 \mathrm{mM} \mathrm{EGTA})$, at increasing protein concentration, protein structures on the SLB transitioned from particles (50 nM) to rings $(1 \mu \mathrm{M})$, and from rings to patches $(3 \mu \mathrm{M})$ (SI Appendix, Fig. S5 and S6; Table S1). The sensitivity of the multimeric structures to protein concentration in our AFM analysis, and the variation in the morphology of these multimers, suggest structural plasticity in the multimerization process. In comparison, in $1 \mathrm{mM}$ free $\mathrm{Ca}^{2+}$, rings and patches still formed, but both classes of multimers

194 formed at lower protein concentrations as compared to the $\mathrm{Ca}^{2+}$-free condition (SI Appendix, Fig.

195 S6; Table S1). The effect of $\mathrm{Ca}^{2+}$ was also consistent across a range of $\mathrm{Ca}^{2+}$ concentrations (SI 196 Appendix, Fig. S7A). The above findings show that all forms of syt1 self-assembly: particles, rings and patches, can occur in the absence of $\mathrm{Ca}^{2+}$, and that addition of $\mathrm{Ca}^{2+}$ facilitates multimerization.

198 To further confirm this $\mathrm{Ca}^{2+}$-dependent enhancement, we tested a syt1 $\mathrm{Ca}^{2+}$ binding mutant, syt $1^{4 \mathrm{~N}}(80-421)$, in which two acidic $\mathrm{Ca}^{2+}$ ligands in each C2-domain were mutated to neutral residues, thus abolishing $\mathrm{Ca}^{2+}$ binding activity $(39,40)$. Ring-like structures were still observed,

201 but $\mathrm{Ca}^{2+}$ failed to enhance further assembly (SI Appendix, Fig. S7B). The precise mechanism by 202 which $\mathrm{Ca}^{2+}$ promotes syt1 self-association is not yet known, but likely involves conformational changes that alter the relative orientation of its tandem C2-domains within the multimer (15). In 
204

205

addition, $\mathrm{Ca}^{2+}$ might also facilitate self-assembly by increasing the local syt1 concentration on the bilayer.

To examine the influence of the SLB phospholipid composition on syt1 self-association, we omitted PS and $\mathrm{PIP}_{2}$ in our protein-lipid interaction tests of syt1(80-421). We found that anionic phospholipids were required for syt1 multimers to assemble on the bilayer (SI Appendix, Fig. S8A). Next, to validate that phospholipid binding promotes syt1 self-association, we deposited syt1(80421) onto a bare mica surface (lipid free), and again studied its morphology under aqueous conditions (SI Appendix, Fig. S8B). When no lipid was present, syt1(80-421) molecules appear as dispersed particles with similar dimensions in both EGTA and $\mathrm{Ca}^{2+}$ conditions.

\section{Lysine residues in the juxtamembrane linker of syt1 are essential for large ring formation} on supported lipid bilayers

As described above, our DLS and EM analysis revealed that syt1 multimerization, in response to lipid binding, is governed by the Juxta $\mathrm{K}$ region. We revisited all the syt 1 mutants characterized by DLS to assess their respective impacts on multimerization on phospholipid bilayers. The WT and mutant syt1(80-421) structures that were analyzed by AFM and the associated lateral height profiles are shown in SI Appendix, Fig. S9-S13. Our AFM analysis found the K326,327A, F349A and R398,399Q mutations have no effect on the formation of large multimeric structures, similar to the DLS result (Fig. 2C). However, also in agreement with our DLS and EM results, we found that neutralizing the juxtamembrane lysine residues (Juxta K) dramatically disrupted the formation of large rings on the SLBs (Fig. 2C). Interestingly, although no large multimeric structures were present with $1 \mu \mathrm{M}$ Juxta $\mathrm{K}$ in $0.5 \mathrm{mM}$ EGTA, smaller $\sim 30 \mathrm{~nm}$ ring structures were formed in the presence of $1 \mathrm{mM}$ free $\mathrm{Ca}^{2+}$ (Fig. $2 \mathrm{C}$ and SI Appendix, Fig. S13). The distinct diameters of the small and large rings (Fig. 2D) suggest that the two populations of syt1(80-421) multimers form by different mechanisms. Notably, the smaller rings have dimensions that are comparable to the rings reported by Rothman, Krishnakumar, Volynski and 
colleagues $(28,32,35,36)$. In stark contrast, however, the small rings observed by AFM in aqueous buffer only appeared in the presence of $\mathrm{Ca}^{2+}$, whereas the rings observed by negative stain EM were dispersed by $\mathrm{Ca}^{2+}(32)$.

\section{Concurrent mutations in two distinct regions of syt1 abolish self-association}

In an effort to further disrupt syt1 self-association (small rings), we analyzed all of the above C2B mutations in a Juxta $\mathrm{K}$ mutant background. Remarkably, only featureless particles were observed in the AFM images of Juxta K + K326,327A, Juxta K + F349A, and Juxta K + R398,399Q (Fig. 2C). These findings reveal that syt1 multimerization is regulated by a complex interplay between the juxtamembrane region and the C2B-domain of syt1, with the juxtamembrane region serving as the primary determinant. These data suggest that the $\mathrm{C} 2 \mathrm{~B}$ mutations may have had subtle effects on self-association that escaped detection when analyzed in an otherwise WT background (Fig. 2C).

\section{Syt1 self-association mutants are targeted to synapses with the correct topology}

Having established that the complete cytoplasmic domain of syt1 forms homo-multimers under aqueous conditions on lipid bilayers, we sought to determine the functional relevance of this interaction by conducting cell-based experiments. We first determined whether each of the mutants described above were properly targeted to SVs in cultured syt1 KO mouse hippocampal neurons. Floxed syt1 was disrupted using Cre recombinase, followed by re-expression of WT or each mutant form of the protein using lentiviral transduction; expression was monitored via immunoblot (Fig. 3A and SI Appendix, Fig. S14; we note that juxtamembrane lysine mutations (Juxta K) reduced the mobility of both recombinant and neuronally expressed syt1 on SDS-PAGE gels). We observed that WT and each mutant form of syt1 were highly co-localized with the SV marker synaptophysin (Fig. 3B and 3C). Notably, the syt1 Juxta K mutant lentiviral expression construct preserved the WT K80 and K81 residues directly following the TMD to ensure proper 
syt1 topology (SI Appendix, Fig. S2) (41). To further confirm targeting to SVs, we conducted pHluorin experiments and found that upon stimulation and exocytosis, all constructs rescued the reduction in the time-to-peak that is characteristic of syt1 KO neurons (19) (Fig. 3D and 3E). Moreover, all but the F349A mutant rescued the kinetic defect in endocytosis that occurs in the KO (19) (Fig. 3F). The unexpected inability of F349A to rescue SV recycling will be addressed in a future study. In summary, these findings demonstrate that all constructs are targeted to SVs with the same topology as the WT protein. However, the low time resolution of the pHluorin measurements sharply limits what can be learned about excitation-secretion coupling, so we next turned to high-speed glutamate imaging and electrophysiology experiments to address the impact of the self-association mutants on evoked and spontaneous neurotransmitter release.

\section{Syt1 self-association is essential for driving and synchronizing evoked SV exocytosis}

In the next series of experiments, we used iGluSnFR (42) to monitor the evoked release of glutamate from SVs, triggered by single action potentials, in WT, syt1 KO, and syt1 KO neurons rescued with each of the constructs detailed above. From the raw traces (Fig. 4A), and from histograms that were created by binning the peak iGluSnFR signal $\left(\Delta F / F_{0}\right)$ versus time (Fig. 4B),

268 it was evident that loss of syt1 abolished rapid synchronous release; only slow asynchronous release was detected. Fast release was completely rescued by WT syt1 and the F349A mutant.

270 In contrast, the Juxta K mutant, alone and combined with the F349A mutant, only partially rescued

271 fast release (Fig. 4A and 4B). Examination of the average traces showed that expression of the

272 Juxta $\mathrm{K}$ mutant resulted in a $63 \pm 1.7 \%$ reduction in the peak amplitude of the iGluSnFR signal, 273 and this was exacerbated by adding the F349A mutation, resulting a $79 \pm 1.7 \%$ reduction in the 274 signal (Fig. 4C, 4D and SI Appendix, Table S2). To visualize the influence that these mutations 275 have on the balance of synchronous vs asynchronous release, we plotted the normalized 276 cumulative frequency distributions for WT, and each mutant, as a function of time. From this 277 analysis, the ability of syt1 to synchronize release is readily apparent (Fig. 5A); the synchronous 
fraction of total release observed over the image series was then extracted and is plotted in Fig. 5B. Substitution of F349 slightly reduced synchronization, but this did not reach significance in our experiments (but see ref. (37)). However, the Juxta K mutations, which strongly affected

281 homo-multimerization, resulted in a marked reduction in the ability of syt1 to synchronize release.

282 Moreover, this effect was even greater when the Juxta K mutant also included the F349A mutation 283 (Fig. 5). Hence, there is a correlation between the ability of mutations to impair syt1 self284 association and to reduce and desynchronize evoked release.

Careful inspection of the iGluSnFR signals, before an action potential was delivered, 287 indicated that some of the syt1 mutations also affected basal fusion rates (Fig. 5A). Indeed, it is 288 well documented that under resting conditions, syt1 serves as a fusion clamp that inhibits 289 spontaneous release $(13-15,43,44)$. We therefore turned to electrophysiological analysis of the 290 spontaneous (i.e. mini) fusion rates in syt1 KO neurons that expressed each construct (Fig. 6). 291 We focused on inhibitory GABAergic minis (mIPSCs) as they are more reliant on syt1 than are 292 glutamatergic minis (45) (Fig. 6). These experiments revealed yet another correlation: mutations 293 that impair syt1 self-association also result in concomitant increases in spontaneous fusion rates 294 (Fig. 6A and 6B). More specifically, F349A partially disrupted the ability of syt1 to clamp minis, as previously reported (37), while the Juxta K mutant had an even stronger effect; mutating both regions almost completely abolished the clamping activity of syt1 (Fig. 6A and 6B). The mini amplitudes were unchanged across all conditions (Fig. 6C). 

22). Indeed, density gradient fractionation (24) and co-immunoprecipitation (46) experiments support the notion that syt1 oligomerizes in the presence of detergent. However, the stoichiometry and structure of these oligomers, as well as the effect of $\mathrm{Ca}^{2+}$ ions and phospholipids on selfassociation, remain unresolved issues. Hence, whether oligomerization impacts syt1 function remains unclear. It is therefore crucial to address the ability of syt 1 to homo-multimerize on lipid bilayers, in the absence of detergent, under physiologically relevant conditions. In the current study, we first addressed this by conducting DLS, EM and AFM measurements in a reconstituted syt $1 \cdot$ membrane systems, under detergent-free aqueous conditions. We observed that the complete cytoplasmic domain of syt1 does in fact form multimers on membranes under relatively native conditions. Importantly, anionic phospholipids are an essential cofactor for self-association (Fig. 1C and SI Appendix, Fig. S8).

We emphasize that our in vitro experiments utilized the complete cytoplasmic domain of syt1, residues $80-421$, which includes the entire juxtamembrane linker between the TMD and the C2A domain. Residues 80-95 have largely been overlooked in previous studies examining syt1 function, but two studies suggested that this region might be important for function. In one report,

317 a peptide corresponding to residues $80-98$ of syt 1 reduced neurotransmitter release, potentially by inhibiting syt1-membrane interactions (47). Also, as mentioned above, the juxtamembrane linker was reported to promote membrane binding and to mediate syt1 glycine zipper interactions (38). Strikingly, we did not observe rings or other multimeric structures when using a shorter

321 fragment that began at residue 96 (unpublished observations); this fragment lacks the 322 juxtamembrane lysine patch. These observations might seem to be at odds with the fact that rings 323 on lipid monolayers were initially observed by EM with a syt1 C2AB domain that lacked the entire 324 juxtamembrane linker (143-421) (32), but in that study, ring formation required low ionic strength 325 buffers (5 - $15 \mathrm{mM} \mathrm{KCl})$ and $40 \%$ PS. Indeed, syt1 self-association is highly dependent on the 326 salt concentration of the buffer (29) and supraphysiological PS promotes calcium-independent 
membrane binding (48). Subsequent EM studies continued to include a low salt step during sample processing; however, it was later determined that the juxtamembrane linker could stabilize ring formation (36) after buffer exchange, from $5 \mathrm{mM}$ to $100 \mathrm{mM} \mathrm{KCl}$, prior to imaging. In short, both mutagenesis and truncation experiments demonstrate that the juxtamembrane linker is required for the cytoplasmic domain of syt1 to form homo-multimers at physiological ionic strength.

Under our aqueous conditions, syt1(80-421) formed large ring-like structures, with diameters $\sim 180 \mathrm{~nm}$, on lipid bilayers; in contrast, previous reports of negative stain EM analysis revealed rings that were $17-45 \mathrm{~nm}$ in diameter (32). Another striking difference was that in our AFM experiments $\mathrm{Ca}^{2+}$ promoted self-assembly, while in the EM studies, $\mathrm{Ca}^{2+}$ dissolved the rings. Interestingly, Wang et al. (2017) also found that $\mathrm{Ca}^{2+}$ stabilized syt1 rings that had assembled in solution after binding a variety of polyanionic compounds. Differences in experimental conditions and sample handling steps, in EM versus AFM experiments, are likely to underlie the observed differences in multimeric structure and regulation. Specifically, AFM facilitates imaging of syt1 on lipid bilayers in aqueous buffer; EM yields greater resolution but involves extensive sample preparation and images are obtained on monolayers under vacuum. At present, we believe that the multimeric structures observed in our system are distinct from the previous reports of syt1 rings.

Interestingly, after neutralizing the juxtamembrane lysines, we observed small rings by AFM that were comparable to the size of the rings that were observed via EM. This may reflect the fact that the majority of the EM studies were, again, conducted using a truncated form of syt1 $(143-421)$ that lacked the crucial juxtamembrane lysine-rich segment $(28,32,35,37)$. We went on to examine all three mutations (K326,327A (27, 34), F349A (28) and R398,399Q (29)) that have been implicated in oligomerization activity mediated by the $\mathrm{C} 2 \mathrm{~B}$ domain. None of these substitutions abolished self-association by themselves, but our AFM imaging found that each set of mutations completely disrupted the self-association in the Juxta K mutant background (Fig. 2C). 
352 We therefore conclude that the juxtamembrane linker and the C2B domain of syt1 both contribute

353 to a complex multimerization mechanism. The formation of large and small rings thus appears to

354 involve somewhat distinct structural elements; the precise experimental conditions might 355 determine which element dominates.

We took advantage of the mutations that impaired syt1 self-association in vitro, under relatively physiological aqueous conditions, and conducted functional assays in neurons. Deletion of the entire juxtamembrane linker has been shown to disrupt syt1 function, perhaps by altering the ability of the C2-domains to engage effectors (49). However, the role of the Juxta $\mathrm{K}$ region in SV exocytosis had not been previously explored via a mutagenesis approach. We first established that each mutant was efficiently targeted to SVs with proper topology, and then conducted physiology experiments that revealed a clear correlation between the ability of the mutations to disrupt multimerization activity with their ability to disrupt the clamping activity of syt 1 , resulting in higher rates of spontaneous SV release. Moreover, impairment of syt1 self-association was also correlated with reductions in peak glutamate release, and the desynchronization of this release, in response to single action potentials. Interestingly, a recent study using a reconstituted vesicle fusion assay proposed that syt 1 must first clamp fusion in order to become subsequently responsive to $\mathrm{Ca}^{2+}(50)$. Together with our in vitro studies, these cell-based findings strongly suggest that syt 1 must assemble into multimers, mainly via its juxtamembrane lysine-rich patch, but also with contributions from the $\mathrm{C} 2 \mathrm{~B}$ domain, in order to inhibit spontaneous release and to drive rapid and efficient evoked release. 
govern syt1 self-association on lipid bilayers. Although the precise structural arrangement and oligomeric status of syt1 on SVs is still unknown, our data suggest that the $\sim 15$ molecules of syt1 per SV would likely multimerize with one-another in vivo. We also note that $\sim 20-25 \%$ of neuronal syt1 resides in the plasma membrane of presynaptic boutons (51-53), and this pool could also potentially play a role in exocytosis by forming large (>15 copies) oligomers. Regardless, the in vitro experiments reported here demonstrate that highly purified syt1 self-associates under native conditions and these assays provided a means to monitor the disruption of this activity via mutagenesis to, in turn, guide functional experiments. Again, our pHluorin experiments largely rule-out a role for juxtamembrane-mediated oligomerization in endocytosis (Fig. 3F), but we demonstrate that syt1 self-association clearly plays a role in clamping spontaneous release (Fig. 6) and in determining the extent and synchronization of evoked SV exocytosis (Fig. 4 and 5). regulate SV fusion. It seems likely that Juxta K-mediated multimerization of syt1 on the surface of SVs would, in addition to facilitating $\mathrm{C} 2 \mathrm{~B}-\mathrm{C} 2 \mathrm{~B}$ interactions, influence how the C2-domains engage with binding partners on the pre-synaptic plasma membrane. For example, syt1 oligomerization might serve to 'order' SNARE proteins around the fusion pore, via direct physical interactions with t-SNAREs $(10,11,39)$. Oligomerization could also play additional roles in the regulation of release by: adding mass to the fusion complex to drive pore dilation, orienting the C2-domains of syt1 to mediate its distinct effects on spontaneous and evoked release (15), allowing copies of syt1 to functionally cooperate with one another via direct physical interactions

397 (22), or allowing groups of syt1 molecules to penetrate bilayers (29) and rearrange the 398 phospholipids, as an ensemble, to drive fusion pore transitions. These issues will be addressed 399 in future studies and will be facilitated by the robust AFM approach that, for the first time, allows 400 the study of syt1 self-association under physiological conditions.

\section{METHODS}




\section{Reagents.}

403 1,2-dioleoyl-sn-glycero-3-phosphocholine (PC), 1,2-dioleoyl-sn-glycero-3-phospho-l-serine (PS),

404 1,2-dioleoyl-sn-glycero-3-phosphoethanolamine $\quad(P E), \quad$ 1-palmitoyl-2-oleoyl-sn-glycero-3-

405 phospho-(1'-rac-glycerol) (PG) and 1,2-dioleoyl-sn-glycero-3-phospho-(1'-myo-inositol-4',5'-

406 bisphosphate) $\left(\mathrm{PI}(4,5) \mathrm{P}_{2}\right)$ were obtained from Avanti Polar Lipids. HEPES was from Fisher

407 Scientific, calcium chloride solution (1.0 M) was from Fluka Analytics, and all other chemicals were from Sigma-Aldrich.

\section{Recombinant proteins.}

410 Recombinant rat syt1 was purified from E. coli (BL21) as an N-terminally tagged his6-SUMO

411 fusion protein. Protein expression was induced by the addition of $200 \mu \mathrm{M}$ isopropyl $\beta$-d-1-

412 thiogalactopyranoside (IPTG) when the $\mathrm{OD}_{600}$ of the culture reached $0.6-0.8$. Bacterial pellets

413 were lysed by sonication in $50 \mathrm{mM}$ Tris, $300 \mathrm{mM} \mathrm{NaCl}, 5 \%$ glycerol, $5 \mathrm{mM}$ 2-mercaptoethanol,

$4141 \%$ Triton $\mathrm{X}-100(\mathrm{pH} 7.4)$ plus a protease inhibitor cocktail (Roche). The samples were also

415 incubated with RNase and DNase $(10 \mu \mathrm{g} / \mathrm{ml})$ to prevent nucleic acid mediated aggregation(54).

416 Insoluble material was removed by centrifugation at $4000 \mathrm{rpm}$ for 15 minutes, and the supernatant

417 was incubated with Ni-NTA agarose, followed by washing the beads with $50 \mathrm{mM}$ Tris, $1 \mathrm{M} \mathrm{NaCl}$,

$4185 \%$ glycerol $(\mathrm{pH}$ 7.4). Protein was liberated from the Ni-NTA agarose by overnight incubation with

$4190.5 \mu \mathrm{M}$ recombinant SENP2 protease. A final FPLC purification was performed by running the 420 samples through a Superdex 200 Increase 10/300 GL column in 25 mM HEPES, $300 \mathrm{mM} \mathrm{NaCl}$,

$4215 \%$ glycerol, 5 mM 2-mercaptoethanol ( $\mathrm{pH}$ 7.4). Samples were subjected to SDS-PAGE and 422 protein concentration was determined by staining with Coomassie blue, using BSA as a standard.

\section{DLS analysis}

424 DLS of syt1(80-421) was performed using a DynaPro NanoStar Dynamic Light Scattering 425 instrument (Wyatt Technology). The syt1(80-421) protein $(2 \mu \mathrm{M})$ was suspended in $25 \mathrm{mM}$ 
426 HEPES, $100 \mathrm{mM} \mathrm{KCl}$, with and without $100 \mu \mathrm{M}$ 1,2-dihexanoyl-sn-glycero-3-phospho-L-serine

427 (6:0 PS) and average diameter distributions were determined. Each of the WT and mutant 428 syt1(80-421) samples were analyzed in triplicate with consistent results. The DLS samples were 429 then blinded and imaged by EM as previously described (55).

$430 \quad$ AFM imaging

431 Thirty $\mu$ l of liposomes (1mM stock solution; PC/PS/PIP $2,72: 25: 3$ or PC/PS, 80:20, extruded with 432 a $100 \mathrm{~nm}$ filter) were suspended in $270 \mu$ limaging buffer (25 mM HEPES, pH 7.4, $150 \mathrm{mM}$ 433 potassium gluconate, $0.5 \mathrm{mM} \mathrm{EGTA}$ ) with or without $1.5 \mathrm{mM} \mathrm{CaCl}_{2}$, and deposited onto freshly 434 cleaved mica (20 mm diameter discs). After incubating for $30 \mathrm{~min}$, the sample was rinsed with $435150 \mu \mathrm{l}$ of the same buffer six times, using a pipette, to remove unabsorbed material; substrate 436 was covered by buffer at all times. Purified syt1(80-421) was suspended in the same buffer and 437 added to the sample. After a 20 -min or 6-hour incubation period, the sample was again rinsed 438 repeatedly to remove unbound protein, and the final sample volume was adjusted to $300 \mu$ l. AFM 439 imaging was carried out with a Agilent 5500 Scanning Probe Microscope in AAC mode with silicon 440 nitride probes (FastScan-D, Bruker or BL-AC40TS, Oxford Instruments). Images were captured 441 with minimum imaging force at a scan rate of $2 \mathrm{~Hz}$ with 512 lines per area. Data analysis was

442 performed with Picolmage 5.1.1. Particle volume was determined using the peak/dip volume tool 443 (within the Picolmage software) line-by-line. Ring structure diameter was defined as the distance 444 between the two highest points on each side of a cross-section across the imaged structure. 445 Protein coverage on lipid bilayers were determined by setting the height threshold to anything one $446 \mathrm{~nm}$ or more above the bilayer surface.

\section{Giant unilamellar vesicle and supported lipid bilayer microscopy}

448 Giant unilamellar vesicles (GUVs) and rhodamine-PE labeled supported lipid bilayers (SLB) were 449 imaged using a Zeiss 880 Airyscan microscope. GUVs were prepared by drying $15 \mu \mathrm{l}$ of $1 \mathrm{mM}$ 
DOPC/DOPS lipids, plus $0.1 \%$ rhodamine-PE in three locations onto indium tin oxide coated glass

451 slides and then performing electroformation with $10 \mathrm{~Hz}, 4 \mathrm{~V}$ alternating current for two hours in 1

$452 \mathrm{mM}$ HEPES, $200 \mathrm{mM}$ Sucrose solution. The buffer also contained $10 \mu \mathrm{M}$ Alexa-647 dye. The

453 excess dye was removed by filtration. The SLBs were prepared on mica discs as described above,

454 except with the inclusion of $0.1 \%$ rhodamine-PE within the lipid mixture.

\section{Cell Culture}

456 Syt1 floxed neurons were prepared as previously described (14). Briefly, hippocampal neurons

457 were dissected at P0 from syt1 floxed mouse strain (Quadros et al., 2017), trypsinized (Corning;

458 25-053-Cl), triturated, and plated on glass coverslips (Warner instruments; 64-0734 (CS-18R17))

459 coated with poly-D-lysine (Thermofisher; ICN10269491) and EHS laminin (Thermofisher;

460 23017015). Neurons were grown for at least 14 days in Neurobasal-A (Thermofisher; 10888-022)

461 medium supplemented with B-27 (2\% Thermofisher; 17504001), Glutamax (2 mM Gibco;

462 35050061), and pen/strep before experiments. For virus preparation, HEK293T cells (ATCC)

463 were cultured following ATCC guidelines and were tested for mycoplasma contamination using

464 the Universal Mycoplasma Detection Kit (ATCC; 30-1012K), and validated as HEK293T cells

465 using Short Tandem Repeat profiling by ATCC (ATCC; 135-XV) within the last year.

466 Lentivirus production and use

467 Lentivirus production was performed as described previously (14). Syt1 expressing lentiviral 468 constructs were subcloned into the FUGW transfer plasmid (FUGW was a gift from David 469 Baltimore (Addgene plasmid \# 14883) (56). Our lab has previously modified this construct, 470 replacing the ubiquitin promoter with the human synapsin I promoter (57). Lentivirus expressing 471 Cre was added to neuronal cultures at day one in-vitro (DIV), iGluSnFR and pHluorin constructs 472 were also added at 1 DIV. Syt1 constructs were added at 5 DIV.

\section{Plasmid construction for lentiviral expression}


474 All plasmids, unless otherwise noted, were constructed using our lab's modified lentivirus

475 backbone of choice derived from FUGW. The glutamate sensor is the same as used previously

476 (14). The pHluorin construct used here was subcloned into our modified FUGW transfer vector

477 from the original vGlut1-pHluorin construct (58). All synaptotagmin 1 constructs were subcloned

478 into our modified FUGW transfer plasmid from their original bacterial expression plasmids. For

479 Cre expression, we used the transfer plasmid pLenti-hSynapsin-CRE-WPRE (pLenti-hSynapsin-

480 CRE-WPRE was a gift from Fan Wang (Addgene plasmid \# 86641) (59).

\section{Immunoblot analysis}

482 Immunoblots were performed as described previously (14). Primary antibodies were: anti-syt1 $483(1: 1000,48)$ (lab stock; mAB 48; RRID:AB_2199314). Secondary antibodies were: goat anti484 mouse IgG2b-HRP (Biorad, M32407; RRID:AB_2536647).

\section{Immunocytochemistry (ICC)}

486 Immunocytochemistry was performed as previously described (14). Primary antibodies were: anti487 syt1 $(1: 100,48)$ (lab stock; mAB 48; RRID:AB_2199314) and anti-synaptophysin (SYP) (1:500) 488 (SySy; 101 004; RRID:AB_1210382). Secondary antibodies used were: goat anti-guinea pig IgG489 Alexa Fluor 546 (1:500) (Thermofisher; A-11074, RRID:AB_2534118) and goat anti-mouse 490 IgG2b-Alexa Fluor 647 (1:500) (Thermofisher; A-21242; RRID:AB_2535811). Images in Fig. 3 491 were acquired on a Zeiss LSM 880 with a $63 \times 1.4$ NA oil immersion objective using the Airyscan 492 super-resolution detector. The same laser and gain settings were used for each condition. Images 493 were deconvolved using automatic Airyscan settings and the same linear brightness and contrast 494 adjustments were applied to all images for figure presentation.

\section{5 iGluSnFR imaging and quantification}


iGluSnFR imaging and quantification was performed as previously described (14), with the

497 following modifications. For single stimuli imaging, 150 frames were collected at 10 ms exposure

498 (1.5 sec total) and a single field stimulus was triggered at half a second after the initial frame.

pHluorin imaging

500

Live cell fluorescent imaging of pHluorin expressing neurons was carried out under the same

501 conditions as iGluSnFR imaging. Briefly, images were acquired on an Olympus IX83 inverted microscope equipped with a cellTIRF 4Line excitation system using an Olympus 60x/1.49 Apo N objective and an Orca Flash4.0 CMOS camera (Hamamatsu Photonics). This microscope runs Metamorph software with Olympus 7.8.6.0 acquisition software from Molecular Devices. Imaging media was extracellular fluid $(\mathrm{ECF})$, with $2 \mathrm{mM} \mathrm{CaCl}_{2}$. Single image planes were acquired with 500 ms exposure using a white OLED with standard GFP filters. Images were collected once a second for 3 minutes. A stimulation train was started 9 seconds into imaging. The train (200 stimuli in 10 seconds $(20 \mathrm{~Hz})$ ) were triggered by a Grass SD9 stimulator through platinum parallel wires attached to a field stimulation chamber (Warner Instruments; RC-49MFSH). All biosensor imaging experiments were performed at $32-34{ }^{\circ} \mathrm{C}$. Environment was controlled by a Tokai incubation controller and chamber.

\section{Colocalization quantification}

513 Colocalization was measured as described previously (14), using Fiji for ImageJ and Just Another 514 Colocalization Plugin (JACoP) (60).

\section{Electrophysiology}

516 Miniature inhibitory postsynaptic currents (mIPSCs) were recorded using a Multiclamp 700B

517 amplifier (Molecular Devices) and analyzed as previously described (45). Briefly, syt1 KO 518 hippocampal neurons expressing WT, Juxta K, F349A or Juxta K + F349A at DIV 14-19 were 519 transferred to a recording chamber with a bath solution containing the following (in $\mathrm{mM}$ ): $128 \mathrm{NaCl}$, 


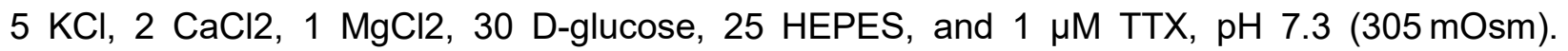

521 Borosilicate glass pipettes (Sutter Instruments) were pulled by a Dual-stage glass micropipette 522 puller (Narishige) and filled with an internal solution contained (in mM): $130 \mathrm{KCl}, 1 \mathrm{EGTA}, 10$

523 HEPES, 2 ATP, 0.3 GTP, 5 QX-314 (Abcam), and 5 sodium phosphocreatine, pH 7.35

524 (295 mOsm). mIPSCs were pharmacologically isolated by bath applying D-AP5 (50 $\mu \mathrm{M}, \mathrm{Abcam})$

525 and CNQX (20 $\mathrm{MM}$, Abcam) and acquired using a Digidata 1440B analog-to-digital converters

526 (Molecular Devices) and Clampex 10 software (Molecular Devices) at $10 \mathrm{kHz}$. Neurons were held

527 at $-70 \mathrm{mV}$. All cells were equilibrated for $\sim 1 \mathrm{~min}$ after break-in before recordings started. Series

528 resistance was compensated, and traces were discarded if the access resistance exceeded 15

$529 \mathrm{M} \Omega$ for the entire duration. The collected miniature events were detected in Clampfit 11.1

530 (Molecular Devices) using a template matching search.

\section{Statistics}

532 Values from analysis and number of trials $(n)$ for each experiment are listed in the figure legends.

533 All analysis was done using GraphPad Prism 7.04 (GraphPad Software Inc).

\section{ACKNOWLEDGMENTS}

535 We thank the members of the Chapman Lab for providing helpful discussions, Julie Morasch for 536 assistance with AFM experiments, and Noah D. Miller for assistance with plasmid and lentivirus 537 preparation. This work was funded by the National Institutes of Health (grants MH061876 and 538 NS097362 to E.R.C.). E.R.C. is an Investigator of the Howard Hughes Medical Institute.

\section{COMPETING FINANCIAL INTERESTS}

540 The authors declare no competing financial interests.

\section{LITERATURE CITED}

542 1. G. J. Augustine, How does calcium trigger neurotransmitter release? Current Opinion in $543 \quad$ Neurobiology 11, 320-326 (2001). 
544 2. B. Katz, The release of neural transmitter substances. Liverpool University Press, 5-39 545 (1969).

546 3. R. Miledi, Transmitter release induced by injection of calcium-ions into nerve terminals. Proceedings of the Royal Society Series B-Biological Sciences 183, 421-425 (1973).

548

549

4. N. Brose, A. G. Petrenko, T. C. Sudhof, R. Jahn, Synaptotagmin: a calcium sensor on the synaptic vesicle surface. Science 256, 1021 (1992).

5. M. Geppert et al., Synaptotagmin-I - A major $\mathrm{Ca}^{2+}$ sensor for transmitter release at a central synapse. Cell 79, 717-727 (1994).

6. M. S. Perin, V. A. Fried, G. A. Mignery, R. Jahn, T. C. Südhof, Phospholipid binding by a synaptic vesicle protein homologous to the regulatory region of protein kinase $C$. Nature 345, 260-263 (1990).

7. J. Bai, W. C. Tucker, E. R. Chapman, PIP2 increases the speed of response of synaptotagmin and steers its membrane-penetration activity toward the plasma membrane. Nature Structural \& Molecular Biology 11, 36-44 (2004).

8. A. Bhalla, W. C. Tucker, E. R. Chapman, Synaptotagmin isoforms couple distinct ranges of $\mathrm{Ca} 2+, \mathrm{Ba} 2+$, and Sr2+ concentration to SNARE-mediated membrane fusion. Molecular Biology of the Cell 16, 4755-4764 (2005).

9. M. M. Bradberry, H. Bao, X. Lou, E. R. Chapman, Phosphatidylinositol 4,5-bisphosphate drives $\mathrm{Ca} 2+-$ independent membrane penetration by the tandem $\mathrm{C} 2$ domain proteins synaptotagmin-1 and Doc2 $\beta$. Journal of Biological Chemistry 294, 10942-10953 (2019).

10. G. Schiavo, G. Stenbeck, J. E. Rothman, T. H. Sollner, Binding of the synaptic vesicle vSNARE, synaptotagmin, to the plasma membrane t-SNARE, SNAP-25, can explain docked vesicles at neurotoxin-treated synapses. Proceedings of the National Academy of Sciences of the United States of America 94, 997-1001 (1997).

11. A. F. Davis et al., Kinetics of synaptotagmin responses to $\mathrm{Ca} 2+$ and assembly with the core SNARE complex onto membranes. Neuron 24, 363-376 (1999).

12. E. R. Chapman, "How does synaptotagmin trigger neurotransmitter release?" in Annual Review of Biochemistry. (Annual Reviews, Palo Alto, 2008), vol. 77, pp. 615-641.

13. J. T. Littleton, M. Stern, M. Perin, H. J. Bellen, Calcium dependence of neurotransmitter release and rate of spontaneous vesicle fusions are altered in Drosophila synaptotagmin mutants. Proceedings of the National Academy of Sciences 91, 10888 (1994).

14. J. D. Vevea, E. R. Chapman, Acute disruption of the synaptic vesicle membrane protein synaptotagmin 1 using knockoff in mouse hippocampal neurons. Elife 9, 24 (2020).

15. H. Bai et al., Different states of synaptotagmin regulate evoked versus spontaneous release. Nature Communications 7, 9 (2016).

16. Z. Wang, H. S. Liu, Y. W. Gu, E. R. Chapman, Reconstituted synaptotagmin I mediates vesicle docking, priming, and fusion. Journal of Cell Biology 195, 1159-1170 (2011). 
594

595

596

597

598

599

600

601

602

603

604

605

606

607

608

609

610

611

612

613

614

615

616

17. N. E. Reist et al., Morphologically docked synaptic vesicles are reduced in synaptotagmin mutants Drosophila. Journal of Neuroscience 18, 7662-7673 (1998).

18. J. T. Littleton et al., synaptotagmin mutants reveal essential functions for the C2B domain in Ca2+-triggered fusion and recycling of synaptic vesicles in vivo. Journal of Neuroscience 21, 1421-1433 (2001).

19. K. Nicholson-Tomishima, T. A. Ryan, Kinetic efficiency of endocytosis at mammalian CNS synapses requires synaptotagmin I. Proceedings of the National Academy of Sciences of the United States of America 101, 16648-16652 (2004).

20. E. M. Jorgensen et al., Defective recycling of synaptic vesicles in synaptotagmin mutants of Caenorhabditis elegans. Nature 378, 196-199 (1995).

21. J. Yao, S. E. Kwon, J. D. Gaffaney, F. M. Dunning, E. R. Chapman, Uncoupling the roles of synaptotagmin I during endo- and exocytosis of synaptic vesicles. Nature Neuroscience 15, 243-249 (2012).

22. M. M. Bradberry et al., Molecular Basis for Synaptotagmin-1-Associated Neurodevelopmental Disorder. Neuron 107, 52-64 (2020).

23. S. Takamori et al., Molecular anatomy of a trafficking organelle. Cell 127, 831-846 (2006).

24. M. S. Perin, N. Brose, R. Jahn, T. C. Südhof, Domain structure of synaptotagmin (p65). Journal of Biological Chemistry 266, 623-629 (1991).

25. E. R. Chapman, S. An, J. M. Edwardson, R. Jahn, A novel function for the second C2 domain of synaptotagmin: Ca-triggered dimerization. Journal of Biological Chemistry 271, 5844-5849 (1996).

26. J. H. Bai, C. A. Earles, J. L. Lewis, E. R. Chapman, Membrane-embedded synaptotagmin penetrates cis or trans target membranes and clusters via a novel mechanism. Journal of Biological Chemistry 275, 25427-25435 (2000).

27. E. R. Chapman, R. C. Desai, A. F. Davis, C. K. Tornehl, Delineation of the oligomerization, $\mathrm{AP}-2$ binding, and synprint binding region of the C2B domain of synaptotagmin. Journal of Biological Chemistry 273, 32966-32972 (1998).

28. O. D. Bello et al., Synaptotagmin oligomerization is essential for calcium control of regulated exocytosis. Proceedings of the National Academy of Sciences 115, E7624 (2018).

29. E. Hui et al., Mechanism and function of synaptotagmin-mediated membrane apposition. Nature Structural \& Molecular Biology 18, 813-821 (2011).

30. C. K. Damer, C. E. Creutz, Calcium-dependent self-association of synaptotagmin I. Journal of Neurochemistry 67, 1661-1668 (1996).

31. J. Ubach et al., The $\mathrm{C} 2 \mathrm{~B}$ domain of synaptotagmin I is a Ca2+-binding module. Biochemistry 40, 5854-5860 (2001). 
624

625

626

627

628

629

630

631

632

633

634

635

636

637

638

639

640

641

642

643

644

645

646

647

648

649

650

651

652

32. J. Wang et al., Calcium sensitive ring-like oligomers formed by synaptotagmin. Proceedings of the National Academy of Sciences 111, 13966 (2014).

33. I. Grass, S. Thiel, S. Höning, V. Haucke, Recognition of a basic AP-2 binding motif within the C2B domain of synaptotagmin is dependent on multimerization. J Biol Chem 279, 54872-54880 (2004).

34. Y. Wu et al., Visualization of synaptotagmin I oligomers assembled onto lipid monolayers. Proceedings of the National Academy of Sciences 100, 2082 (2003).

35. J. Wang et al., Circular oligomerization is an intrinsic property of synaptotagmin. eLife 6, e27441 (2017).

36. M. N. Zanetti et al., Ring-like oligomers of Synaptotagmins and related C2 domain proteins. eLife 5, e17262 (2016).

37. E. Tagliatti et al., Synaptotagmin 1 oligomers clamp and regulate different modes of neurotransmitter release. Proceedings of the National Academy of Sciences of the United States of America 117, 3819-3827 (2020).

38. B. Lu, V. Kiessling, L. K. Tamm, D. S. Cafiso, The juxtamembrane linker of full-length synaptotagmin 1 controls oligomerization and calcium-dependent membrane binding. Journal of Biological Chemistry 289, 22161-22171 (2014).

39. C. A. Earles, J. Bai, P. Wang, E. R. Chapman, The tandem C2 domains of synaptotagmin contain redundant $\mathrm{Ca} 2+$ binding sites that cooperate to engage t-SNAREs and trigger exocytosis. J Cell Biol 154, 1117-1123 (2001).

40. J. M. Mackler, J. A. Drummond, C. A. Loewen, I. M. Robinson, N. E. Reist, The C2B Ca2+binding motif of synaptotagmin is required for synaptic transmission in vivo. Nature 418, 340-344 (2002).

41. Y. Kida, M. Sakaguchi, M. Fukuda, K. Mikoshiba, K. Mihara, Membrane topogenesis of a type I signal-anchor protein, mouse synaptotagmin II, on the endoplasmic reticulum. Journal of Cell Biology 150, 719-729 (2000).

42. J. S. Marvin et al., Stability, affinity, and chromatic variants of the glutamate sensor iGluSnFR. Nature Methods 15, 936-+ (2018).

43. N. A. Courtney, H. Bao, J. S. Briguglio, E. R. Chapman, Synaptotagmin 1 clamps synaptic vesicle fusion in mammalian neurons independent of complexin. Nature Communications 10, 14 (2019).

44. H. S. Liu et al., Linker mutations reveal the complexity of synaptotagmin 1 action during synaptic transmission. Nature Neuroscience 17, 670-+ (2014).

45. N. A. Courtney, J. S. Briguglio, M. M. Bradberry, C. Greer, E. R. Chapman, Excitatory and Inhibitory Neurons Utilize Different Ca2+ Sensors and Sources to Regulate Spontaneous Release. Neuron 98, 977-+ (2018). 
653

654

655

656

657

658

659

660

661

662

663

664

665

666

667

668

669

670

671

672

673

674

675

676

677

678

679

680

681

682

683

684

685

686

687

688

689

46. M. Fukuda, K. Mikoshiba, Distinct self-oligomerization activities of synaptotagmin family. Unique calcium-dependent oligomerization properties of synaptotagmin VII. J Biol Chem 275, 28180-28185 (2000).

47. P. Caccin et al., The synaptotagmin juxtamembrane domain is involved in neuroexocytosis. Febs Open Bio 5, 388-396 (2015).

48. M. Bendahmane et al., The synaptotagmin C2B domain calcium-binding loops modulate the rate of fusion pore expansion. Molecular Biology of the Cell 29, 834-845 (2018).

49. J. Lee, J. T. Littleton, Transmembrane tethering of synaptotagmin to synaptic vesicles controls multiple modes of neurotransmitter release. Proceedings of the National Academy of Sciences of the United States of America 112, 3793-3798 (2015).

50. S. Ramakrishnan, M. Bera, J. Coleman, J. E. Rothman, S. S. Krishnakumar, Synergistic roles of synaptotagmin-1 and complexin in calcium-regulated neuronal exocytosis. Elife 9, 18 (2020).

51. F. Opazo et al., Limited intermixing of synaptic vesicle components upon vesicle recycling. Traffic 11, 800-812 (2010).

52. M. Wienisch, J. Klingauf, Vesicular proteins exocytosed and subsequently retrieved by compensatory endocytosis are nonidentical. Nature Neuroscience 9, 1019-1027 (2006).

53. T. Fernandez-Alfonso, R. Kwan, T. A. Ryan, Synaptic vesicles interchange their membrane proteins with a large surface reservoir during recycling. Neuron 51, 179-186 (2006).

54. C. Liu, Y. Zhang, "Nucleic acid-mediated protein aggregation and assembly" in Advances in Protein Chemistry and Structural Biology, R. Donev, Ed. (Academic Press, 2011), vol. 84, pp. 1-40.

55. Z. Zhang, E. R. Chapman, Programmable Nanodisc Patterning by DNA Origami. Nano Letters 20, 6032-6037 (2020).

56. C. Lois, E. J. Hong, S. Pease, E. J. Brown, D. Baltimore, Germline transmission and tissue-specific expression of transgenes delivered by lentiviral vectors. Science 295, 868872 (2002).

57. S. Kugler, E. Kilic, M. Bahr, Human synapsin 1 gene promoter confers highly neuronspecific long-term transgene expression from an adenoviral vector in the adult rat brain depending on the transduced area. Gene Therapy 10, 337-347 (2003).

58. S. M. Voglmaier et al., Distinct endocytic pathways control the rate and extent of synaptic vesicle protein recycling. Neuron 51, 71-84 (2006).

59. K. Sakurai et al., Capturing and manipulating activated neuronal ensembles with CANE delineates a hypothalamic social-fear circuit. Neuron 92, 739-753 (2016).

60. S. Bolte, F. P. Cordelieres, A guided tour into subcellular colocalization analysis in light microscopy. Journal of Microscopy 224, 213-232 (2006). 
A

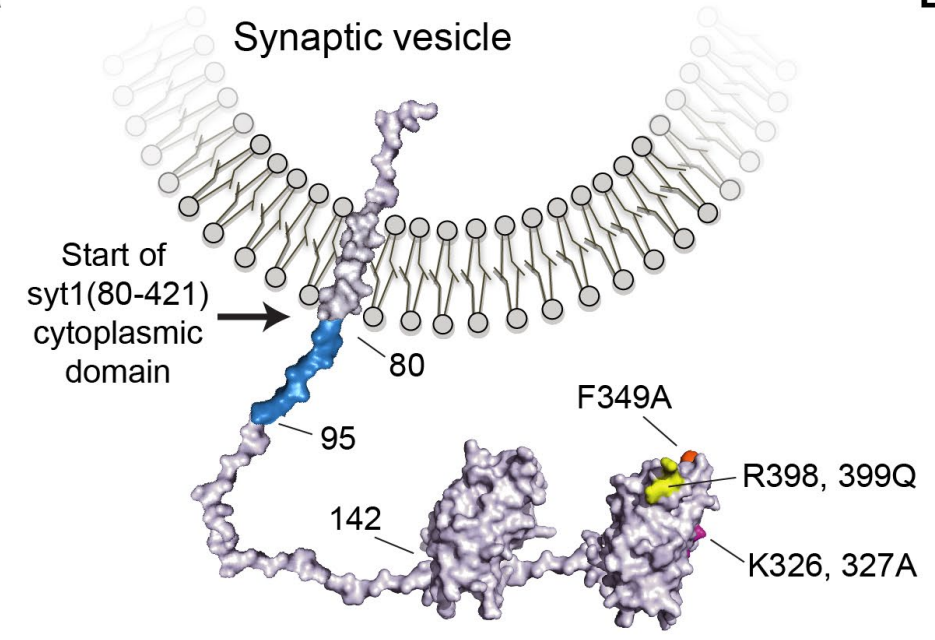

Plasma membrane

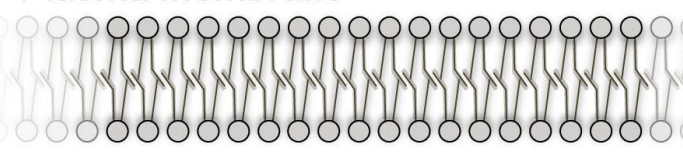

Juxtamembrane poly-lysine segment

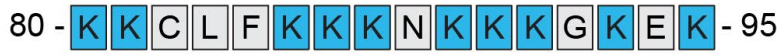

B

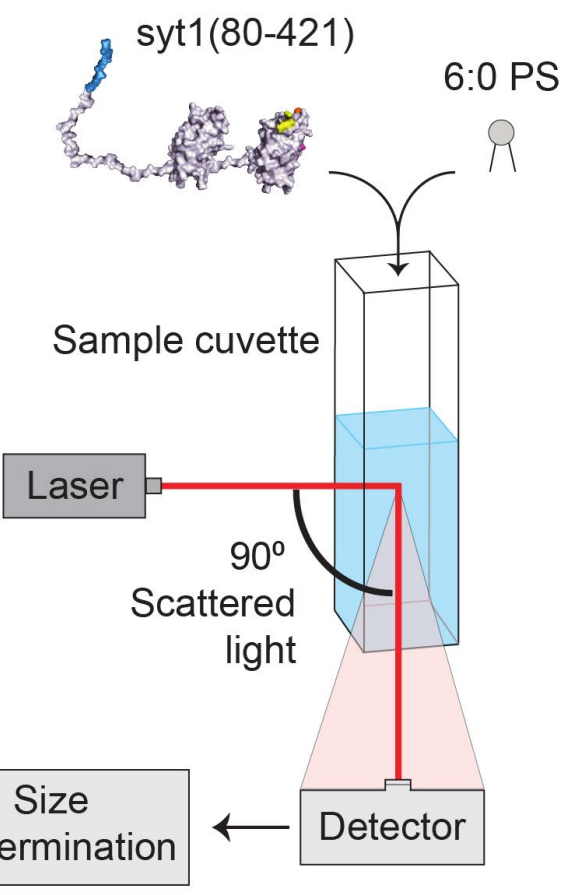

C
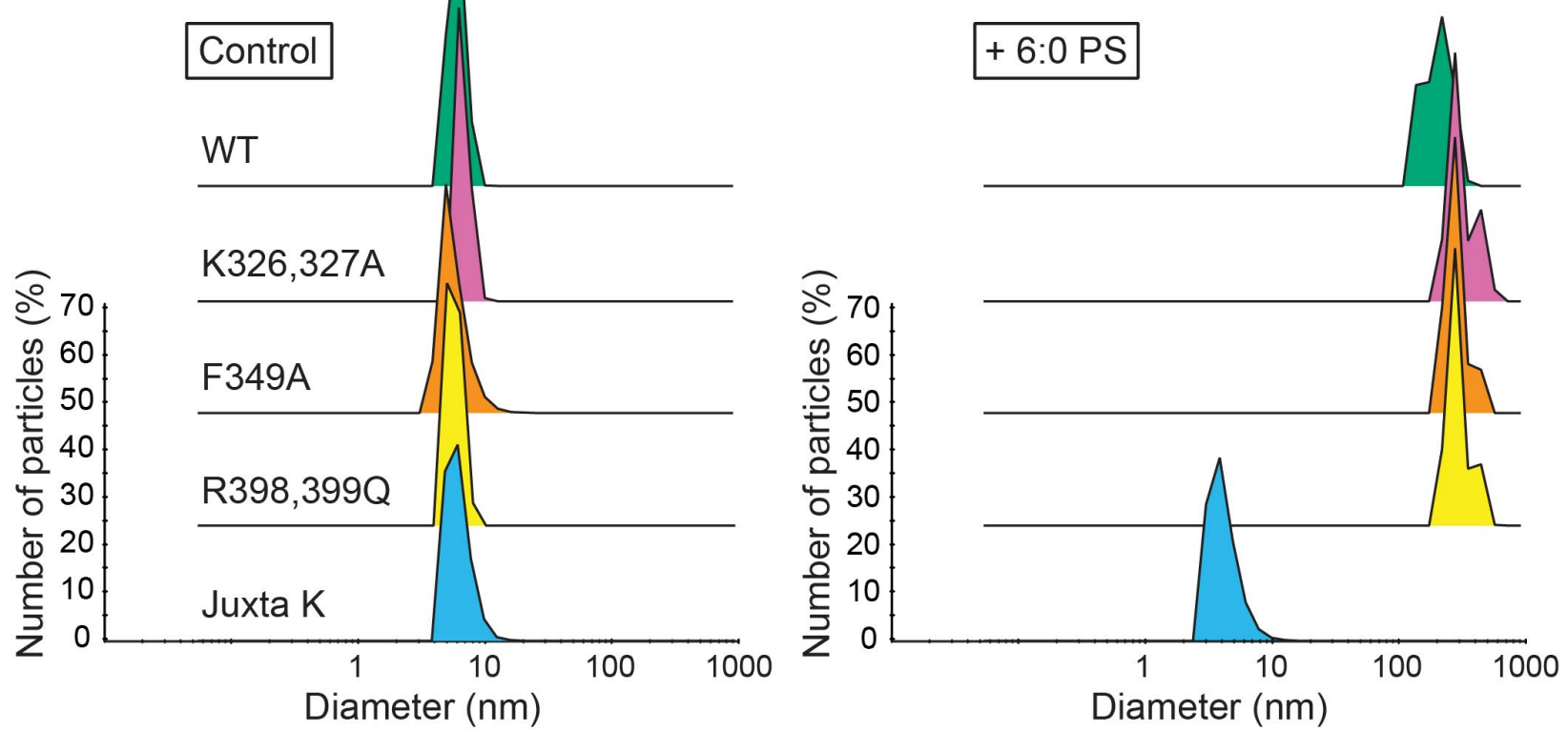

D WT syt1

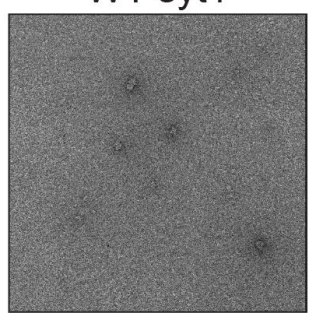

Juxta K

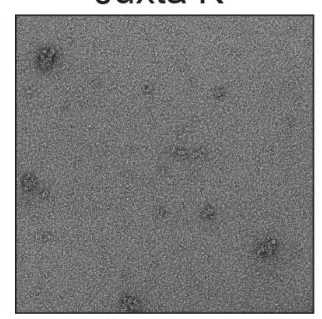

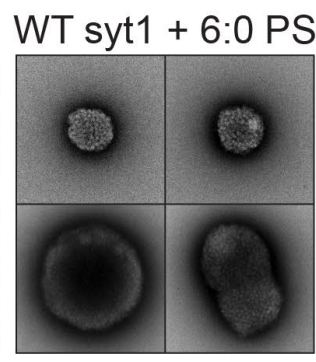

6:0 PS
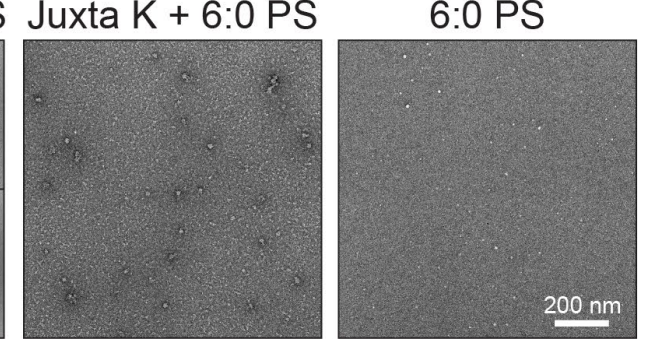
691 Fig. 1. The complete cytoplasmic domain of syt1 forms large oligomers in solution upon addition

692 of 6:0 PS. A) Structure of full-length syt1, embedded in a synaptic vesicle (SV) membrane, with 693 relevant residue annotations. Structures of the C2-domains were derived from RCSB PDB 5t0r 694 (C2A) and PDB 2yoa (C2B); other linker segments, derived from PBD 5w5c, were added. The 695 syt1 oligomerization mutations tested in this study are color coded as follows: K326,327A, 696 magenta; F349A, orange; and R398,399Q, yellow. The polylysine juxtamembrane region is 697 emphasized in blue. The amino acid sequence (residues 80-95) of the syt1 juxtamembrane region 698 immediately following the transmembrane domain. Lysine residues are shown in blue. B) Cartoon 699 depiction of the DLS assay. C) Representative results of the DLS analysis showing the diameter 700 of $2 \mu \mathrm{M}$ WT and mutant syt1(80-421) with and without the addition of $100 \mu \mathrm{M}$ 6:0 PS. Average 701 diameters for each condition, performed in triplicate, are shown in Table 1. D) Representative EM 702 images of WT and Juxta K mutant syt1(80-421) samples that were analyzed by DLS, with and 703 without the addition of 6:0 PS. Scale bar represents $200 \mathrm{~nm}$. 
A

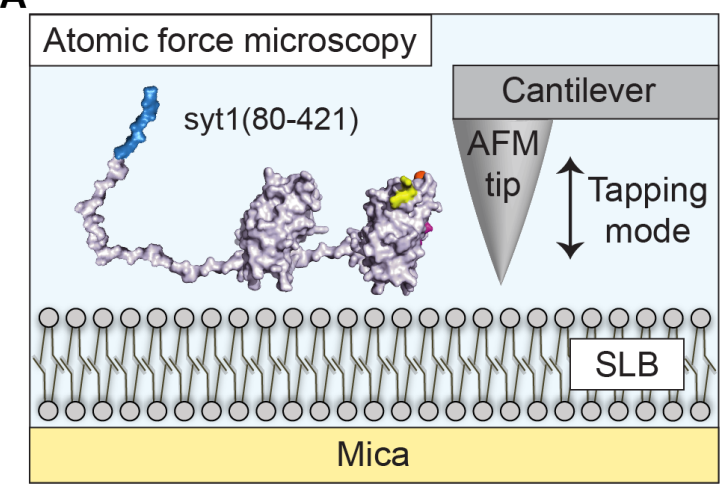

C
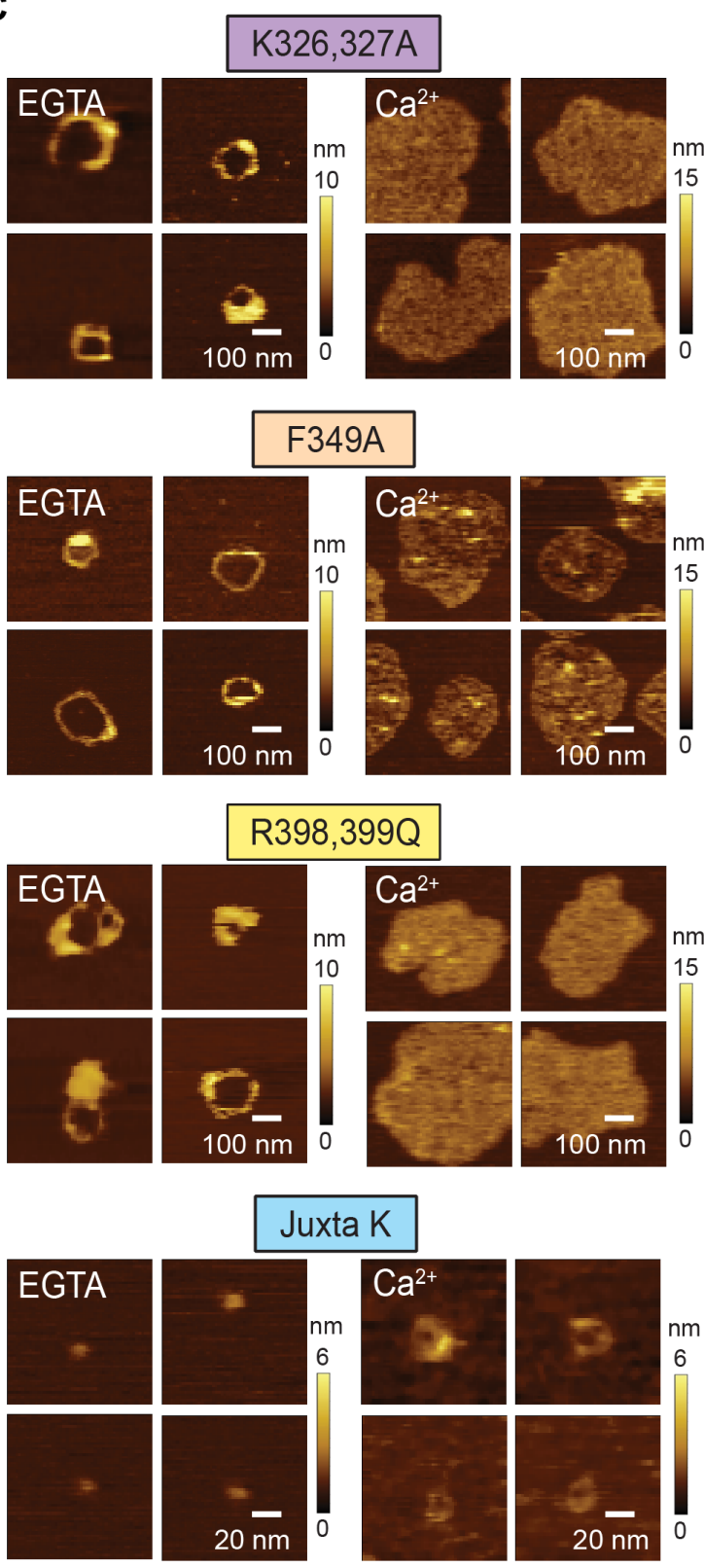

B
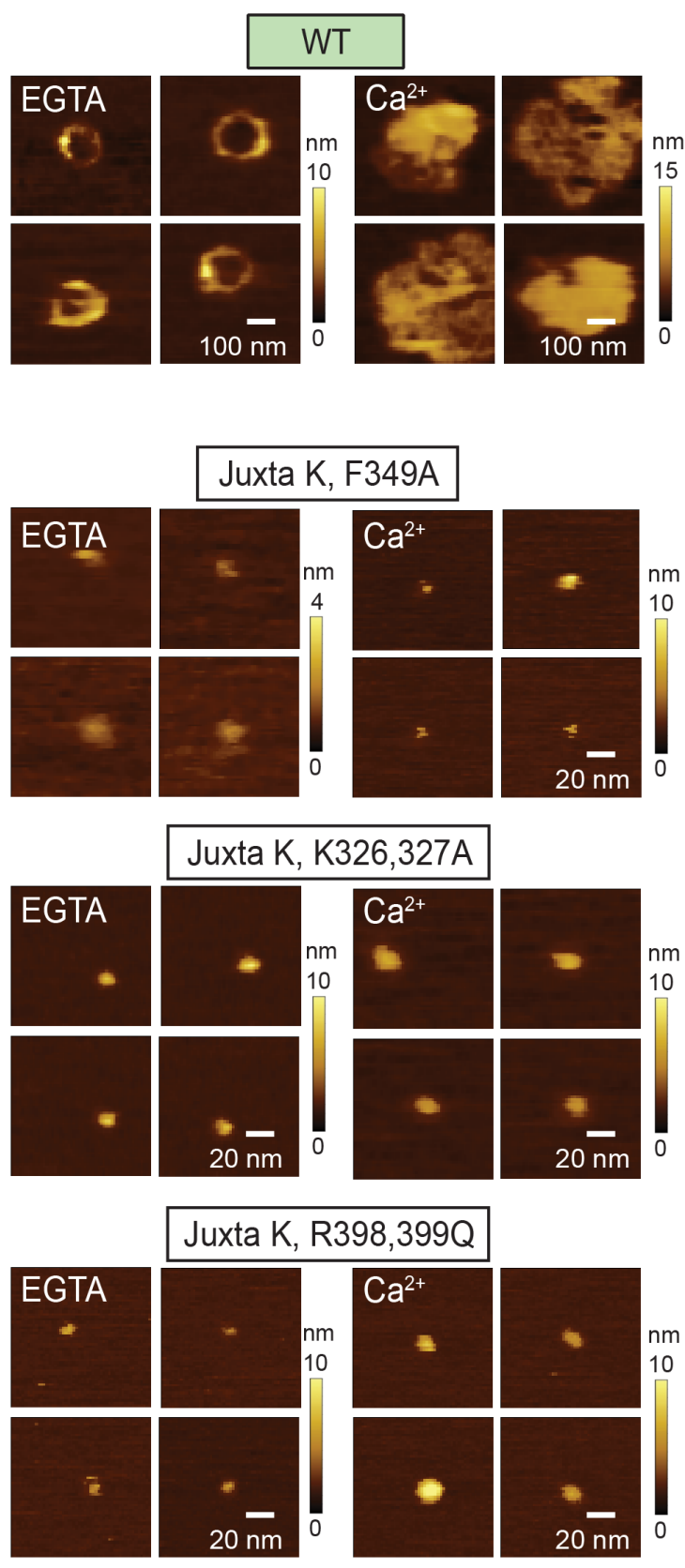

D

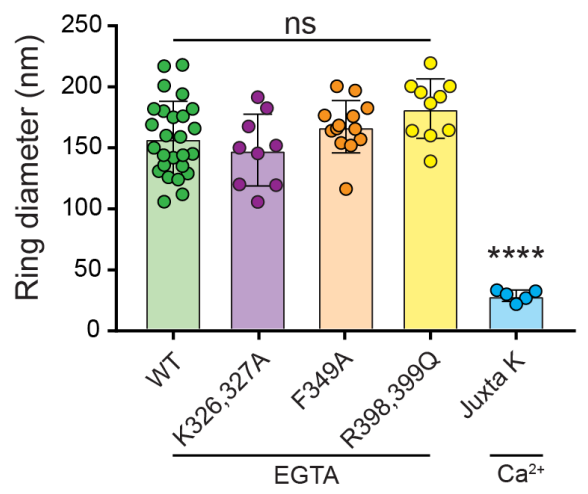


Fig. 2. AFM imaging of WT and mutant syt1(80-421) on supported lipid bilayers. A) Cartoon depiction of the AFM experimental conditions. B) Representative AFM topographical images of 1 $\mu$ M WT syt1(80-421) on supported lipid bilayers (72\% DOPC, 25\% DOPS, $3 \% \mathrm{PIP}_{2}$ ) in the presence $(1 \mathrm{mM})$ and absence $(0.5 \mathrm{mM} \mathrm{EGTA})$ of $\left.\mathrm{Ca}^{2+} . \mathbf{C}\right)$ Representative AFM topographical 710 images of the syt1(80-421) mutants under the same conditions as the WT samples. The protein 711 concentration was $1 \mu \mathrm{M}$ in all cases except for the $\mathrm{R} 398,399 \mathrm{Q}$ sample, where a concentration of $712300 \mathrm{nM}$ was used to better visualize ring formation. Pixel size: left column $9.8 \mathrm{~nm} / \mathrm{px}$, right column $7132.0 \mathrm{~nm} / \mathrm{px}$. D) Ring diameters for WT syt1(80-421) (green), K326,327A (purple), F349A (orange), 714 and $\mathrm{R} 398,399 \mathrm{Q}$ (yellow) in the absence of $\mathrm{Ca}^{2+}$, and of Juxta K (blue) in the presence of $\mathrm{Ca}^{2+}$. 715 For the Juxta $\mathrm{K}$ sample in $\mathrm{Ca}^{2+}, 41.6 \%$ of the observed structures were rings, while the remainder were particles. Additional C2B mutations, in the Juxta $\mathrm{K}$ background, abolished ring formation so

717 these samples were excluded from ring analysis. ${ }^{* * *}$ Denotes p-values $<0.0001$ for Juxta $\mathrm{K}$, 718 compared to all other conditions. For WT, F349A, K326,327A, R398,399Q and Juxta K, the 719 number of rings measured were: $26,13,9,10$ and 5 for each condition from 3, 2, 2, 2, and 2 sets of analyses using independent SLBs, respectively. Error bars represent SEM. 
A

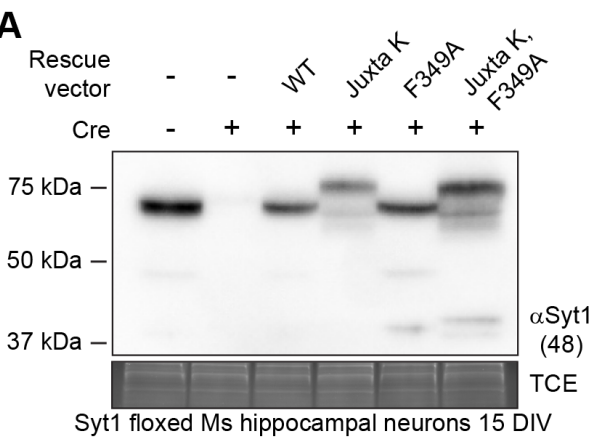

C

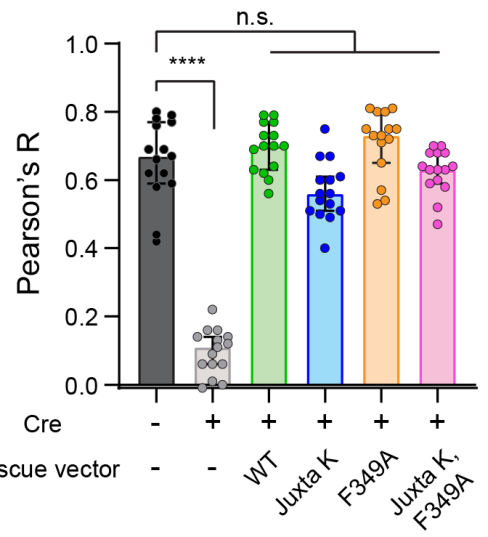

D

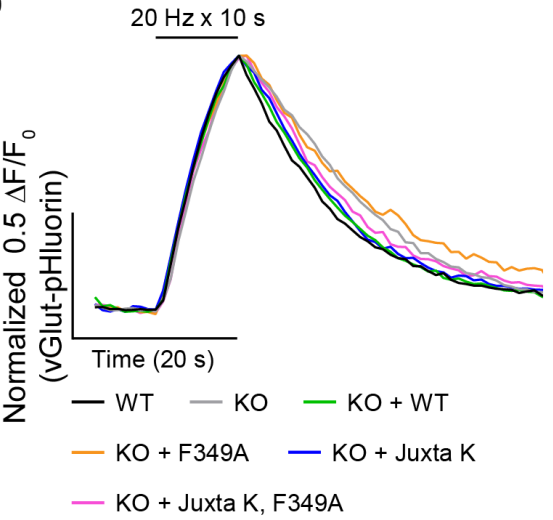

B

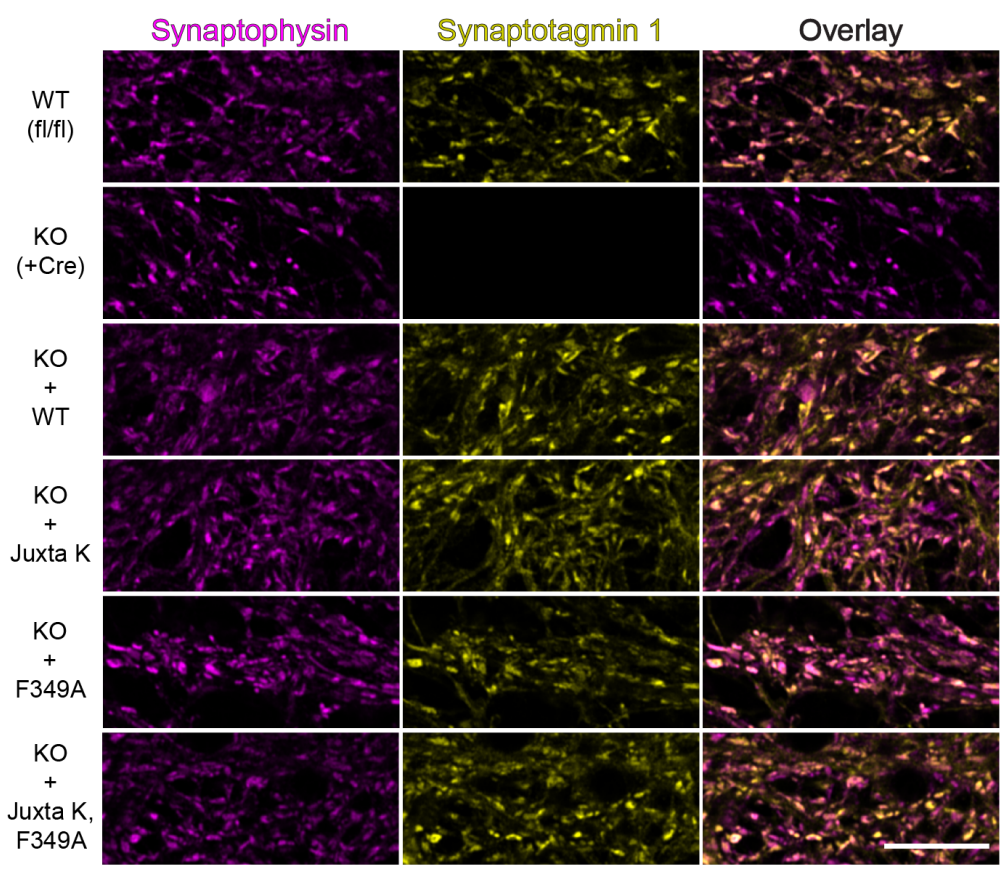

E

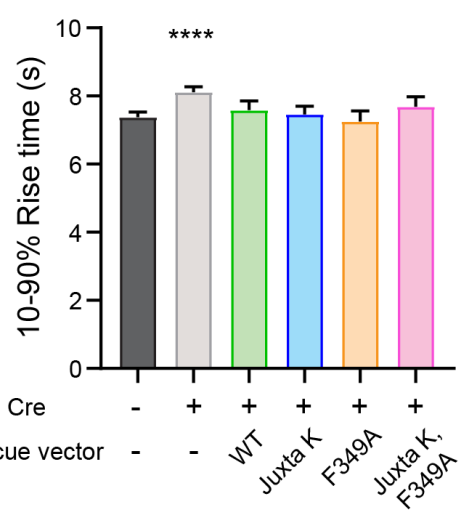

$\mathbf{F}$

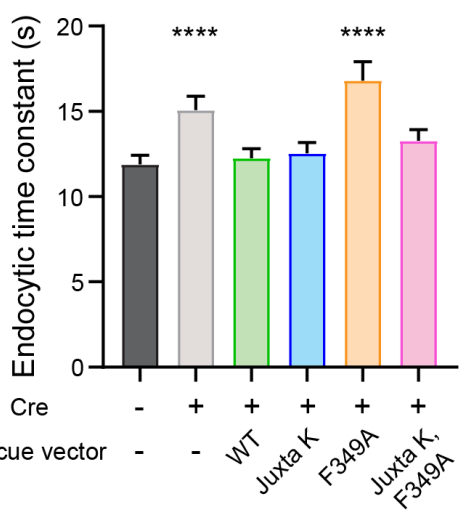

723 Fig. 3. WT and mutant syt1 constructs are efficiently targeted to SVs. A) Representative anti-syt1

724 immunoblots of WT, syt1 KO (generated using a Cre virus), and KO neurons expressing WT syt1,

725 Juxta K mutant syt1, F349A mutant syt1, and the double Juxta K + F349A mutant syt1, in mouse

726 hippocampal neurons at 15 DIV. B) Representative super-resolution fluorescent ICC images from

727 mouse hippocampal neurons at 21 DIV. Images of WT, syt1 KO (+Cre), and KO neurons

728 expressing WT syt1, Juxta K mutant syt1, F349A mutant syt1, and the double Juxta K + F349A

729 mutant syt1, stained with anti-synaptophysin (magenta) and anti-syt1 (yellow) antibodies; in the

730 last column these signals are merged. C) Bar graph of the Pearson's correlation coefficient (PCC) 
731 statistic R. Values were obtained using ROls from whole fields of view and JaCoP for ImageJ (60).

732 Plot of the median Pearson's $\mathrm{R}+/-95 \% \mathrm{Cl} ; \mathrm{n}=15$ for each condition from three trials. These data 733 were not normally distributed; ${ }^{* * *}$ denotes $\mathrm{p}$-value $<0.0001$ between WT and syt $1 \mathrm{KO}$, no other 734 conditions were statistically significantly different from WT using Kruskal-Wallis test with Dunn's 735 correction. Full statistics are included in Fig. 3-source data. D) Partially cropped, averaged, and 736 normalized vGlut1-pHluorin traces from each indicated condition obtained using wide field 737 fluorescence, imaged once a second for 120 seconds. Twenty $\mathrm{Hz}$ field stimulation began at $\mathrm{t}=$ $73810 \mathrm{~s}$ (200 action potentials) in 14 DIV hippocampal mouse neurons. E) vGlut1-pHluorin 10\%-90\% 739 peak rise times were plotted. Values are median with $95 \% \mathrm{Cl} ; 500$ to $700 \mathrm{ROIs}(\mathrm{n})$ were analyzed 740 from three separate trials. These data were not normally distributed' ${ }^{* * * *}$ denotes $p$-value $<0.0001$ 741 between WT and syt1 KO, no other conditions were statistically significantly different from WT 742 using Kruskal-Wallis test with Dunn's correction. F) vGlut1-pHluorin decay times represented as 743 time constants determined from fitting data to a single exponential function. Values are median 744 with $95 \% \mathrm{Cl} ; 500$ to 700 ROls (n) measured from three separate trials. These data were not 745 normally distributed; ${ }^{* * *}$ denotes $p$-value $<0.0001$ between WT and syt1 KO, and WT and F349A 746 mutant rescue, no other conditions were statistically significantly different from WT using Kruskal747 Wallis test with Dunn's correction. 
bioRxiv preprint doi: https://doi.org/10.1101/2021.07.28.454225; this version posted July 29, 2021. The copyright holder for this preprint (which was not certified by peer review) is the author/funder. All rights reserved. No reuse allowed without permission.

A

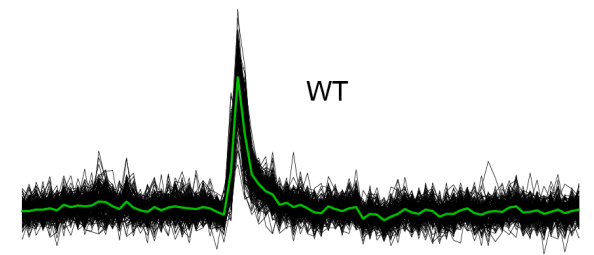

KO
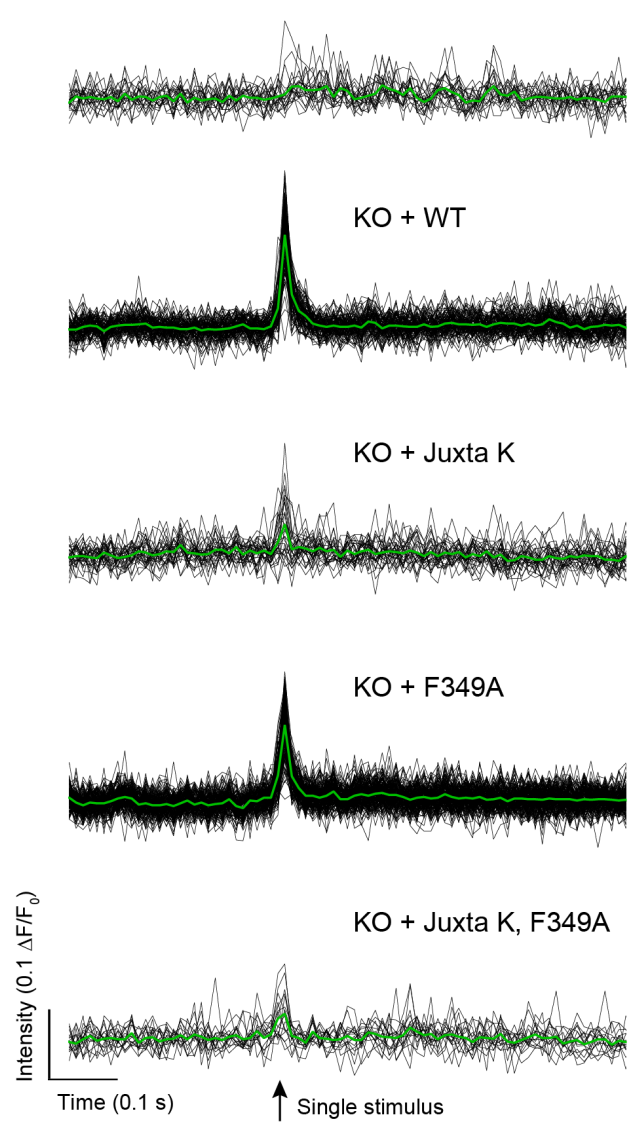

C

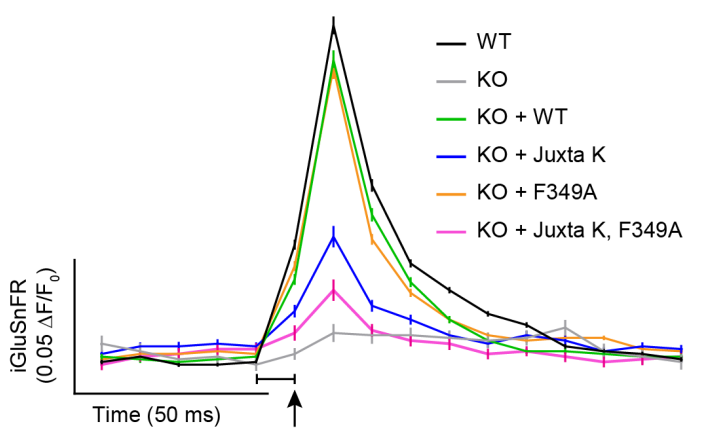

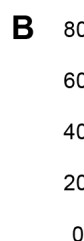
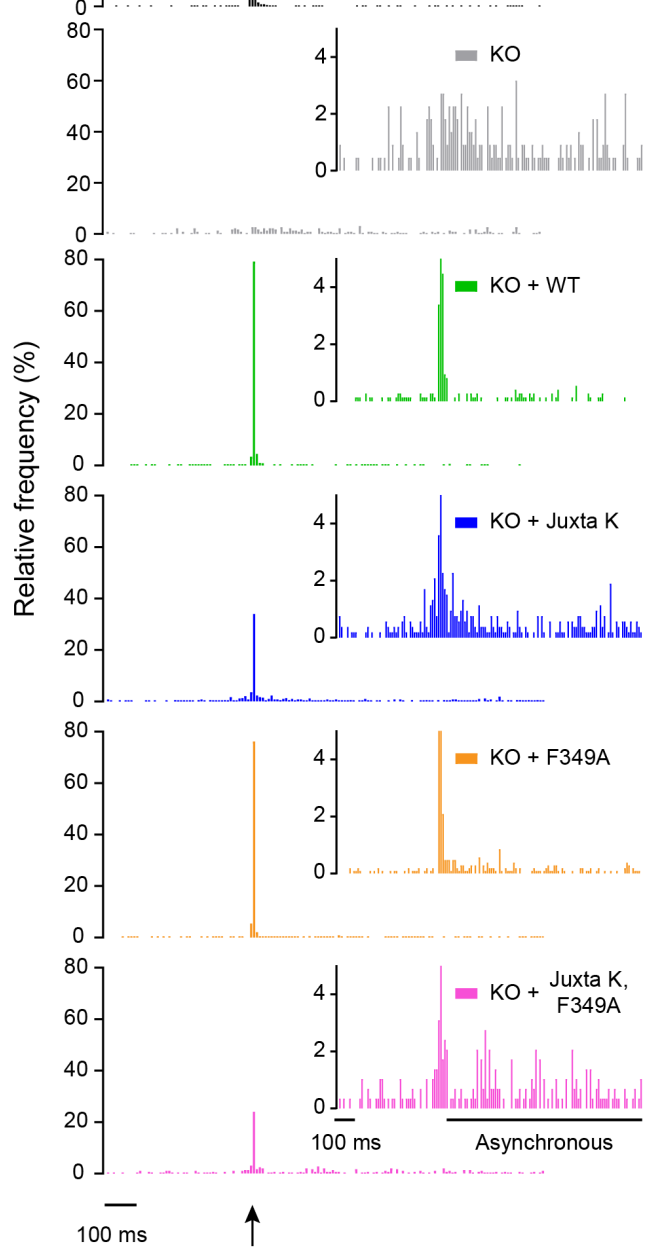

D

Individual iGluSnFR peaks All other comparisons $p<0.001$

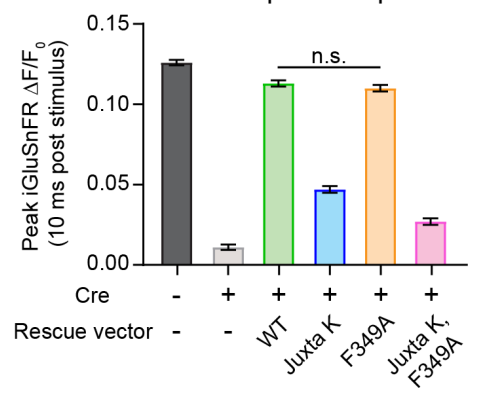


Fig. 4. Lysine residues in the syt1 juxtamembrane linker regulate evoked neurotransmitter release.

A) Representative iGluSnFR traces from one field of view after a single field stimulus (indicated by a black arrow) at $100 \mathrm{~Hz}$. The plots show the iGluSnFR responses from multiple individual

753 ROls (black) and the average responses (green). Note that the $\mathrm{KO}, \mathrm{KO}+$ Juxta $\mathrm{K}$ and $\mathrm{KO}+\mathrm{Juxta}$

$754 \mathrm{~K}, \mathrm{~F} 349 \mathrm{~A}$ conditions resulted in fewer responses after stimulation. B) Histograms of iGluSnFR $755(\Delta \mathrm{F} / \mathrm{F} 0)$ peaks plotted using $10 \mathrm{~ms}$ bins. The timing of the stimulus is indicated with a black arrow.

756 Peaks were binned over the entire $1.5 \mathrm{~s}$ of recording. Conditions were color-coded as follows:

757 WT (black), syt1 KO (grey), KO + WT (green), KO + Juxta K (blue), KO + F349A (orange), KO +

758 Juxta K, F349A (magenta). The histograms include combined data from three independent trials.

759 Y-axis zoom-in histograms of iGluSnFR ( $\Delta$ F/F0) peaks shown as an inset to emphasize the 760 presence of asynchronous release. C) Average traces of iGluSnFR $\triangle F / F 0$ from a single field 761 stimulus, indicated by a black arrow, optically recorded at $100 \mathrm{~Hz}$. Note, the $1 \mathrm{~ms}$ stimulus was 762 applied in the last ms of the frame that is indicated by a black bracket; this causes a small portion 763 of the rise in signal to be captured within that frame. Conditions are labeled in the figure and the 764 same color scheme is used throughout. Briefly, WT (black), syt1 KO (grey), WT syt1 rescue 765 (green), Juxta K mutant rescue (blue), F349A mutant rescue (orange), and the double Juxta K + 766 F349A mutant rescue (magenta). Values are means with 95\% Cl error bars; the number of ROIs 767 analyzed were: WT (1155), syt1 KO (212), WT rescue (748), Juxta K rescue (547), F349A rescue 768 (1128), and double mutant rescue (324), collected from three separate trials. D) Average peak 769 iGluSnFR responses $10 \mathrm{~ms}$ post stimulus from each condition. Quantification of data in panel (C). 770 Values are mean +/- SEM from three independent trials. These data were normally distributed 771 and only WT rescue and F349A mutant rescue were statistically similar, all other groups are 772 different using ordinary ANOVA with Holm Sidak's correction. All comparisons and statistical 773 analysis are provided in Fig. 4-source data. 
775

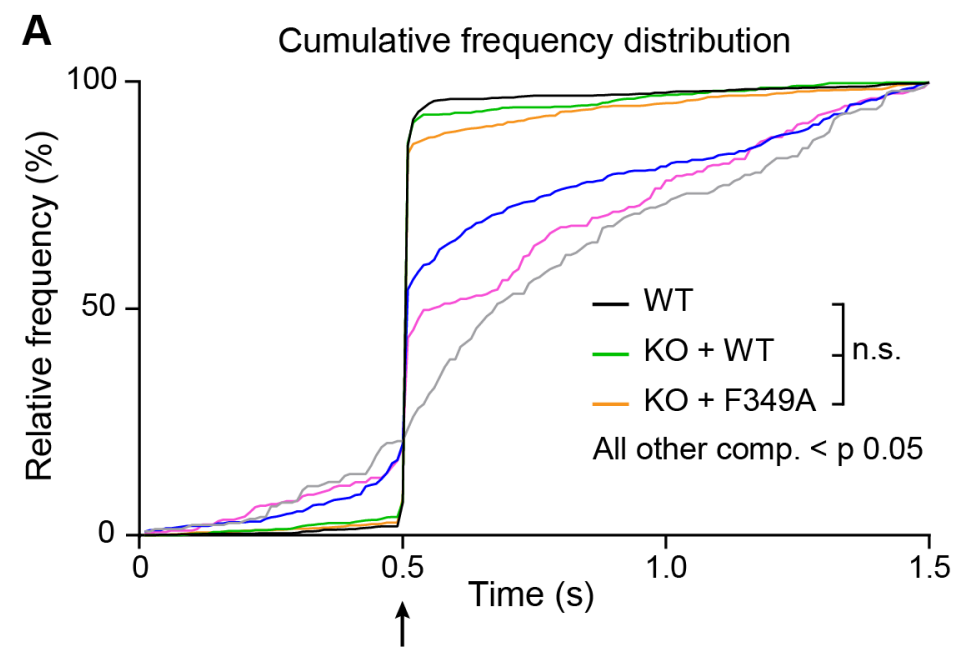

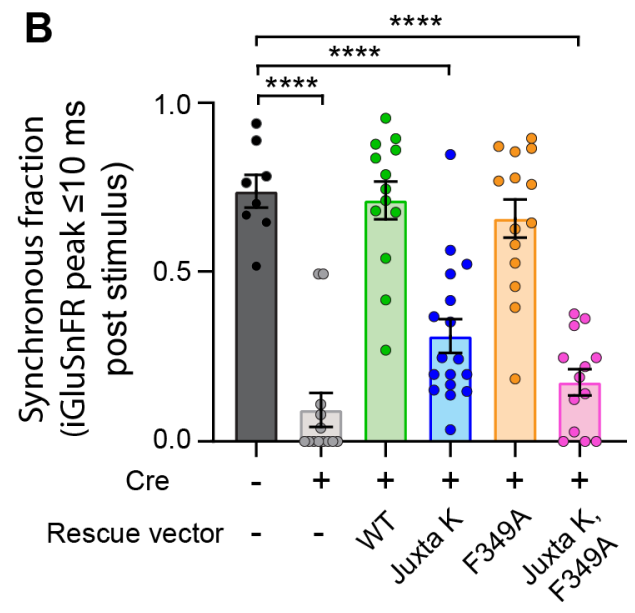

776

777

778

779

780

781

782

783

784

785

786

787

Fig. 5. Lysine residues in the syt1 juxtamembrane linker regulate synchronized neurotransmitter release A) Cumulative frequency distribution of glutamate peaks throughout imaging, analyzed from Fig. 4; these data were not normally distributed. Using Kruskal-Wallis test with Dunn's correction, no difference detected between WT, WT rescue, and F349A mutant rescue. All other comparisons were significantly different with $p<0.05$. Full statistics are included in Fig. 5A-source data. B) Synchronous fraction of each condition quantified. Synchronous release defined as percentage of iGluSnFR $\triangle \mathrm{F} / \mathrm{F} 0$ peaks within $10 \mathrm{~ms}$ following a single stimulus (data are from Fig.

$4 \mathrm{C}$ and $\mathrm{D}$ ) from an entire field of view. Values are mean $+/$ - SEM from three independent trials.

These data were normally distributed, with 8 to 17 field of views for each group; ${ }^{* * * *}$ denotes $p$ value $<0.0001$ between WT and labeled conditions using ANOVA with Holm-Sidak's correction. All comparisons and statistical analysis, provided in Fig. 5B-source data. 
A

KO

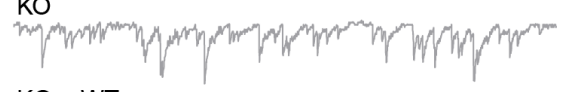

$\mathrm{KO}+\mathrm{WT}$

KO + Juxta K

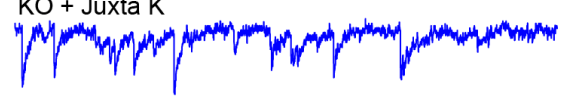

KO + F349A

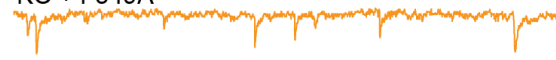

KO + Juxta K, F349A

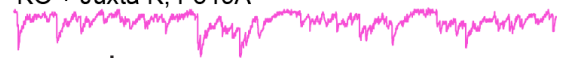
$100 \mathrm{pA}$

$1 \mathrm{~s}$
B

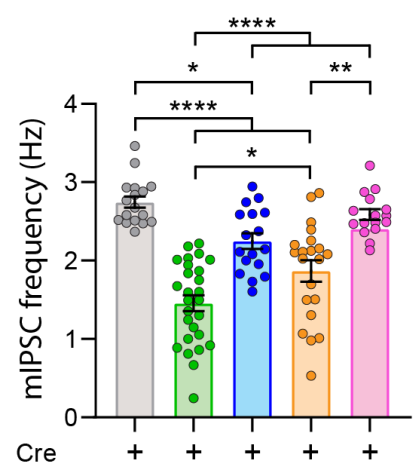

Rescue vector -
C

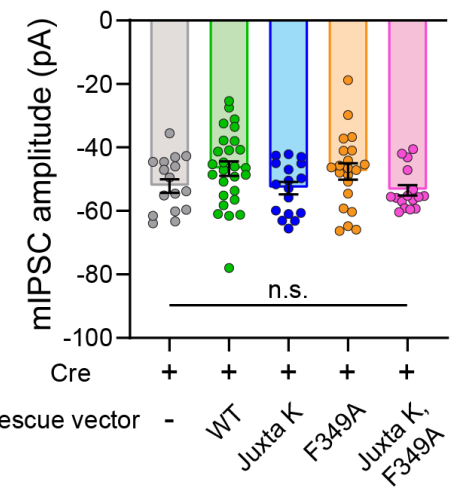

789

790

791

792

793

794

795

796

797

798

799

800

Fig. 6. Lysine residues in the syt1 juxtamembrane linker regulate spontaneous neurotransmitter release. A) Representative traces of mIPSCs from syt1 KO neurons (grey) or syt1 KO expressing WT rescue (green), Juxta K mutant rescue (blue), F349 mutant rescue (orange), and Juxta K + F349A mutant rescue (magenta) syt1. B) Quantification of mIPSC frequencies from neurons expressing the various syt1 constructs. mIPSC frequency was $2.7 \pm 0.07 \mathrm{~Hz}$ in syt1 KO neurons (mean \pm SEM; $n=17), 1.5 \pm 0.10 \mathrm{~Hz}$ in WT syt1 rescued cells $(n=27), 1.9 \pm 0.14 \mathrm{~Hz}$ in F349A rescued cells ( $n=21), 2.2 \pm 0.10 \mathrm{~Hz}$ in Juxta K rescued cells $(n=17)$, and 2.4 $\pm 0.0 .08 \mathrm{~Hz}$ in Juxta K, F349A rescued cells $(n=16)$. C) The quantification of mIPSC amplitudes after expression of the various syt 1 constructs described in panel $A .{ }^{*}$ denotes $p$-values $<0.05$; ${ }^{* *}$ denotes $p$-values $<0.01$ and ${ }^{* * * *}$ denotes p-value $<0.0001$ determined by ANOVA using Tukey's multiple comparisons test. All comparisons and statistical analyses are provided in Fig. 6-source data. 
801 Table 1. Average diameter of syt1(80-421) in solution. DLS analysis of WT and mutant syt1(80-

$802421)(2 \mu \mathrm{M})$ in aqueous buffer, with and without the addition of $100 \mu \mathrm{M} \mathrm{6:0} \mathrm{PS}$. Error represents

803 standard deviation, derived from 3 independent experiments.

\begin{tabular}{|l|l|l|l|l|l|}
\hline & WT & K326,327A & F349A & R398,399Q & Juxta K \\
\hline Control & $6.0 \pm 0.3$ & $5.8 \pm 1.3$ & $5.1 \pm 1.4$ & $5.8 \pm 0.7$ & $5.4 \pm 1.7$ \\
\hline$+\mathbf{6 : 0}$ PS & $215.7 \pm 38.3$ & $366.1 \pm 22.2$ & $321.3 \pm 45.5$ & $373.3 \pm 7.9$ & $5.1 \pm 2.5$ \\
\hline
\end{tabular}

804 